\title{
Fatigue Life Prediction Using Hybrid Prognosis for Structural Health
}

\author{
Monitoring \\ Rajesh Kumar Neerukatti ${ }^{1}$ \\ Arizona State University, Tempe, Arizona 85287 \\ Kuang C. $\mathrm{Liu}^{2}$ \\ Arizona State University, Tempe, Arizona 85287 \\ Narayan Kovvali ${ }^{3}$ \\ Arizona State University, Tempe, Arizona 85287 \\ Aditi Chattopadhyay ${ }^{4}$ \\ Arizona State University, Tempe, Arizona 85287
}

As metallic aircraft components are subject to a variety of in-service loading conditions, predicting their fatigue life has become a critical challenge. To address the failure mode mitigation of aircraft components and at the same time reduce the life cycle costs of aerospace systems, a reliable prognostics framework is essential. In this paper a hybrid prognosis model that accurately predicts the crack growth regime and the residual useful life (RUL) estimate of aluminum components is developed. The methodology integrates physics based modeling with a data-driven approach. Different types of loading conditions such as constant amplitude, random, and overload are investigated. The developed methodology is validated on an Al 2024-T351 lugjoint under fatigue loading conditions. The results indicate that fusing the measured data and physics based models improves the accuracy of prediction compared to a purely data-driven or physics based approach. 
${ }^{1}$ AIAA Student Member - Graduate Research Associate

${ }^{2}$ AIAA Member - Associate Research Scientist

${ }^{3}$ Assistant Research Professor

${ }^{4}$ AIAA Fellow - Ira A. Fulton Professor

\section{Nomenclature}

$$
\begin{array}{ll}
\mathrm{a}_{\mathrm{N}} & =\text { crack length at } \mathrm{N}^{\text {th }} \text { cycle } \\
\mathrm{da} / \mathrm{dN} & =\text { crack growth rate } \\
\mathrm{K} & =\text { stress intensity factor } \\
\mathrm{K}\left(\mathrm{x}, \mathrm{x}_{\mathrm{i}}\right) & =\text { kernel function } \\
\mathrm{M} & =\text { material parameter } \\
\mathrm{P}_{\mathrm{N}} & =\text { load at the } \mathrm{N}^{\text {th }} \text { cycle } \\
\mathrm{S} & =\text { geometric parameter } \\
\mathrm{t}_{\mathrm{n}} & =\text { targets } \\
\mathrm{W}_{\mathrm{i}} & =\mathrm{i}^{\text {th }} \text { weight } \\
\mathrm{W} & =\text { width of the compact tension specimen } \\
y_{*} & =\text { mean of the predicted value } \\
2 \mathrm{~h} & =\text { length of the center cracked plate } \\
& \quad=\text { the lasso estimate } \\
(x) \quad=\text { basis function } \\
\quad=\text { noise } \\
\end{array}
$$




$$
\begin{array}{ll}
{ }_{i} & =\mathrm{i}^{\text {th }} \text { hyperparameter } \\
i^{2} & =\mathrm{i}^{\text {th }} \text { posterior mean } \\
{ }_{*} & =\text { variance of the predicted value }
\end{array}
$$

\section{Introduction}

Accurate estimation of fatigue life of metallic components under complex loading conditions is critical to the safety and reliability of aerospace vehicles. The majority of currently available fatigue life prediction models are deficient in predicting damage under random or flight profile service loads. The inherent inaccuracy of these models is due to the stochastic nature of crack propagation in metallic structures. A significant amount of work has been reported on the development of reliable prognostic frameworks. Current research is primarily focused on datadriven approaches; however, physics based models have also been utilized, the details of which will be presented in the subsequent discussion. Data-driven approaches do not use physics based information, and hence their prediction accuracy is inferior to those based on physics, particularly when there is sparse data or incomplete knowledge. Physics based approaches, on the other hand, are also inadequate in that they have difficulty adapting to variations in response due to material scatter, environmental changes, and other unclassifiable but significant sources of noise. Thus, a hybrid prognostic model, which uses a fusion of physics based model information and measured data, is expected to provide more accurate and reliable information on damage prognosis and residual useful life.

For a specimen under constant amplitude loading, the fatigue crack growth phenomena can be captured using Paris' Law. In contrast, the crack growth caused by variable amplitude loading is characterized by acceleration and retardation effects [1-3], which significantly affect the RUL. Currently there are many physics based models [1-6] to model crack growth with 
acceleration and retardation effects. These models capture the fatigue crack growth phenomena reasonably well under variable loading, but in a deterministic framework. Therefore, they are unable to capture the uncertainty in fatigue crack growth. [7-8].

Ling et al. [9] proposed a method for the integration of structural health monitoring (SHM) with fatigue damage prognosis. The prognosis methodology uses a fracture mechanics based crack growth model, focusing on predictions under uncertainties in the data and model errors using Wheeler's retardation model. Ling et al. [10] also presented a method for predicting the uncertainty in loading by investigating techniques such as rain-flow counting, Markov chain method and autoregressive moving average (ARMA) model. Sankararaman et al. [11] presented a prognosis methodology under variable amplitude multi-axial loading, where an equivalent stress intensity factor (SIF) as a function of the crack length and the loading condition is used. A Gaussian process (GP) regression is then used to calculate the SIF at any crack length. Liu et al. [12] proposed a methodology to calculate the equivalent initial flaw size distribution based on Kitagawa-Takahashi diagram, which is independent of the applied load and only depends on the threshold SIF. Though this method provides good results, it requires the experimentally measured threshold SIF. Hu et al. [13] presented a method for fatigue life prediction using damage mechanics based approach where continuum damage mechanics principles were used to predict the corrosion fatigue crack initiation life of Al 2024-T62 alloy. This method provides good predictions of the damage evolution but it does not provide information on RUL. Grell et al. [14] developed a probabilistic interface for the Air Force Grow (AFGROW) life prediction software, which was demonstrated on compact tension, single edge notched tension, and single lap joint specimens. This study allowed each of the parameters to be modeled as a distribution. However, as this study is based on AFGROW software, which uses analytical formulations for 
predicting the fatigue life, it is limited to simple specimens. Ozaltun et al. [15] developed an energy based fatigue life prediction framework for calculating RUL in gas turbine components. The study shows good results but it is limited to constant amplitude cyclic loading, and does not consider overloads and underloads which are very common for structural components in aircraft.

Several other methods $[3-6,16-21]$ have been proposed for fatigue crack growth modeling. These models are mostly analytical and use a factor to account for geometry of the specimen in their formulation, and therefore have limited applications. Mohanty et al. [22, 23] developed a purely data-driven GP based prognosis framework, combining on-line and off-line information, for damage state estimation and RUL estimation of metallic structural hotspots under complex loading, such as random and Fighter Aircraft Loading STAndard For Fatigue (FALSTAFF) [24, 25]. Mohanty et al. [26] also presented a passive sensing based strain mapping approach for real-time damage state estimation under random loading. In this method, the strains are predicted at any time with new loading information and estimated reference model. The damage states are then evaluated by comparing the predicted and actual strains via correlation analysis. Although these models $[22,23,26]$ provide very accurate results, the accuracy of prediction is dependent on the available training data. In the initial stage, where there is less training data, the prediction is not accurate. However the prediction accuracy increases over time as more training data becomes available [22]. Wang et al. [27] presented a generic probabilistic framework for health prognostics and uncertainty management. The generic framework is formulated using four core elements such as system health index, offline learning scheme, online prediction scheme and uncertainty propagation map. Relevance vector machine (RVM), which is a sparse Bayes learning scheme was used to speed up the data processing as it considers only a few neighboring kernels irrespective of the data size. In the proposed hybrid 
approach, RVM has been used as a regression tool to create a mapping for the SIF as a function of different crack tip locations.

Many studies [28-30] have presented the use of prognostic degradation models for RUL estimation. Gebraeel et al. [28] presented a degradation model to compute the RUL distribution under time-varying environmental conditions by incorporating additional parameters to the degradation models that capture multiple environmental conditions. Bian et al. [29] studied systems that degrade gradually and whose degradation can be monitored using sensor technology. The sensor based degradation signals were modeled by using different techniques such as Brownian motion process, gamma process, etc. The principal objective of their research was to develop a stochastic degradation modeling approach to estimate residual life distributions for components operating in field conditions. Gebraeel et al. [30] developed a degradation modeling framework in which the RUL predictions can be made in the absence of prior knowledge. This was accomplished by utilizing failure time data instead of the sensor-based degradation signals. Bernstein distribution was used to fit failure time values, and the parameters were used to estimate the distributions of stochastic parameters of the initial degradation model. These models [28-30] provide very good results in field situations and can drastically improve the predictive capability under uncertainties. Since the results presented in the current paper utilize data from laboratory experiments collected over a short period of time, it was unnecessary to consider the effects of degradation in the analysis.

The currently available techniques either use a pure physics based approach or a pure data-driven approach for predicting RUL in metallic structures. In this work, we present a hybrid prognosis methodology that uses information from a physics based model integrated with a datadriven approach for damage state prediction and RUL estimation. More details about the 
proposed hybrid approach are discussed in Section II. The basic algorithm is depicted in Fig. 1. An initial prediction of the crack length is conducted based on material characterization. The estimated damage and loading conditions are used to forecast the crack growth behavior, thus leading to an evaluation of the RUL for the specimen. The results can be utilized within a risk assessment framework to decide if detailed inspection, repair or replacement is necessary.

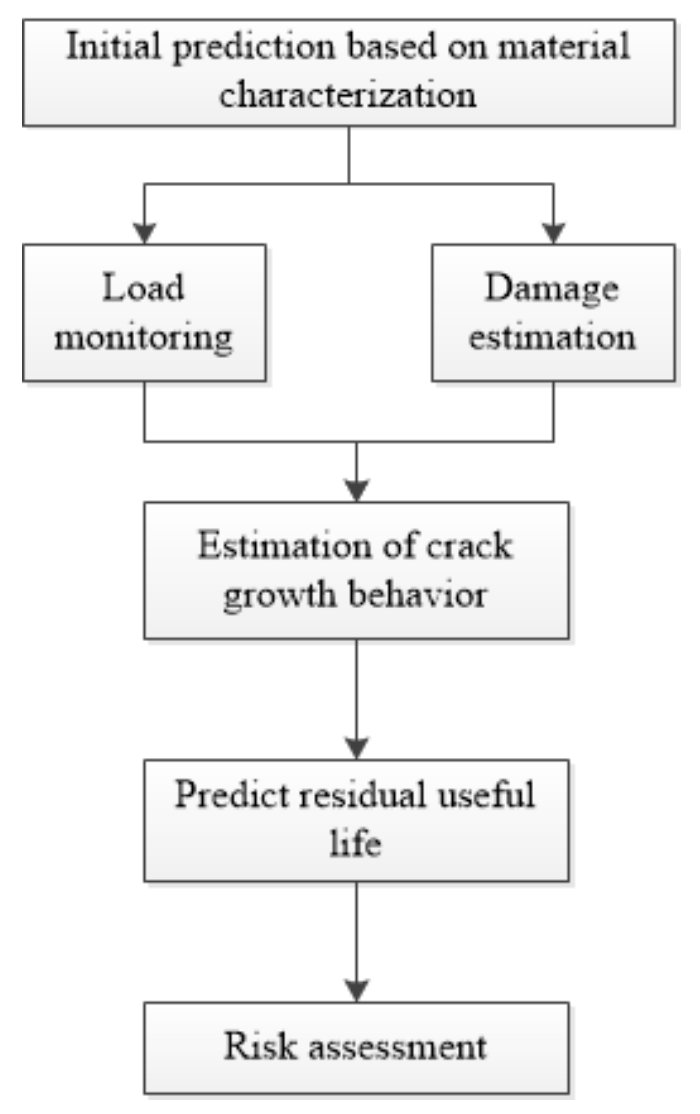

Fig 1. Flowchart of the prognosis algorithm

This paper is organized as follows. In Section II, the hybrid prognosis theory is discussed, including details and parameters of the working model. Section III is devoted to a discussion of results from the methods in Section II.

\section{Hybrid Prognosis Theory}

Fatigue crack growth behavior for a given specimen can be predicted by combining knowledge of the underlying mechanics of crack growth and future loading. The hybrid 
prognosis framework presented in this paper considers simple crack growth models whose behaviors are inferred and updated using data-driven approaches. The combination of physics and data-driven approaches allows for the consideration of proper fracture mechanisms while correcting for material variations and uncertainty in the model parameters using data-driven model updating. Thus, although simple physics models are used, the accuracy of the hybrid framework is greater than those of data-driven and physics based models alone, as shown in the results presented in a later section.

Linear elastic fracture mechanics (LEFM) states that the crack growth rate $(d a / d N)$ is a function of the SIF range ( $\Delta K)$, as shown in Eq. 1.

$\frac{d a}{d N}=f(\Delta K)$

where, $\quad K=K_{\max } K_{\min }$

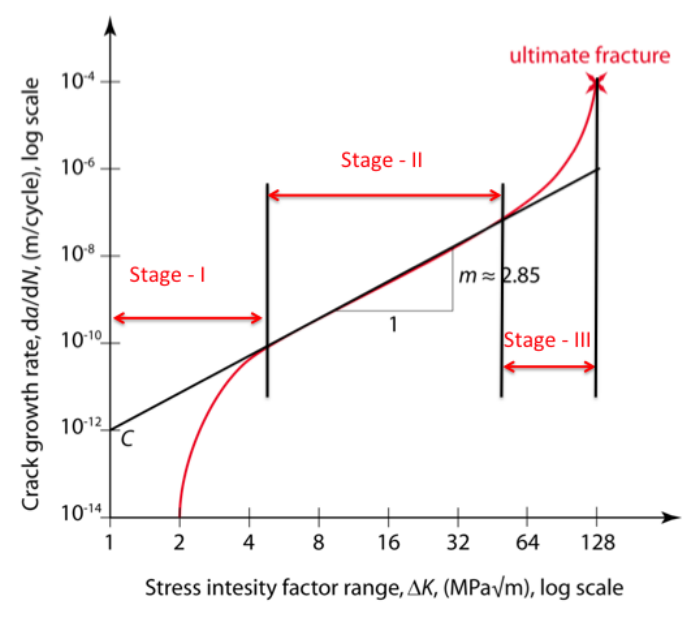

Fig. 2 The relationship between crack growth rate and SIF is highly nonlinear even after log-log transform. There is a stable linear portion, indicated by the black line that is representative of Stage II or sub-critical crack growth.

Most fracture theories $[1,2,31]$ use the above LEFM model with some modifications to account for variability in loading. Due to the exponential nature of crack growth, the relationship between 
crack growth rate and SIF is generally described using log-log transforms. The commonly observed trend showing three critical zones, stages I-III of crack growth, is shown in Fig. 2. Prediction initiation in stage I can often result in large errors with respect to life since cracks can grow on the order of $\mathrm{N}^{3}$ or $\mathrm{N}^{4}$. Typically, prognosis algorithms are applied during stage II or subcritical crack growth and are used to predict ultimate fracture. In some cases, such as for constant amplitude loading, this regime is linear. Models such as Paris' Law are well suited to capture this behavior. For cases such as overloads and underloads, this regime can be highly nonlinear and discontinuous requiring the use of advanced physics models, which are often unavailable. In the hybrid prognosis framework presented here, the exact relationship between crack growth rate and SIF for a given cycle is inferred from the available data based on the assumption of a linear relationship with non-constant coefficients in log space. The coefficients of the linear fit are a function of historical crack growth data, future loading (i.e., overloads/under-loads), and basic material properties and cycles, and are continuously evolving and adapting as more data are available. This is shown in Eq. 2, where $C_{1}$ and $C_{2}$ are coefficient functions, $M$ denotes material parameters, $P$ represents loading, and subscripts $\mathrm{N}-1$ and $\mathrm{N}+1$ denote previous and future loading cycles.

$$
\log \frac{d a}{d N}=C_{1}\left(a_{N-1}, M, P_{N, N+1}, N\right)+C_{2}\left(a_{N-1}, M, P_{N, N+1}, N\right) \log (\Delta K)
$$

Initial estimates for these parameters (i.e., prior to data acquisition) can be obtained through the basic material constants used in Paris' Law, which reduces the crack growth rate estimation to a classical Paris' Law extrapolation. A typical SHM framework will detect, localize and classify damage based on inputs from embedded sensing system [32, 33]. Since the SHM framework provides data on crack length and locations, as well as load monitoring and cycle counting, the coefficients are updated, allowing them to model and capture the nonlinear and discontinuous 
behavior. In order to predict the fatigue crack growth of a specimen, Eq. 2 needs to be formulated in terms of measureable parameters and integrated until ultimate fracture. Therefore, the parameters in Eq. 2 must be written in terms of these data, requiring that SIF is related to known or quantifiable parameters.

We will assume that the SIF can be expressed as a general function

$$
\Delta K_{N}=f\left(a_{N}, P_{N}, S\right)
$$

where, $S$ is a geometric parameter. For simple structures, analytical expressions of SIF are available that describe its dependence on geometry, crack length, and applied load. When an analytical expression is available, it can then be directly substituted into Eq. 1 and the future crack growth can be calculated. However, in the absence of this information, numerical methods must be utilized to provide estimates of SIF. Either method is acceptable and suitable for use in the proposed framework. The developed hybrid approach comprises a physics based formulation to compute the SIF for a given specimen geometry under fatigue loading and a data-driven component for determining the model parameters $\mathrm{C}_{1}$ and $\mathrm{C}_{2}$.

In order to predict RUL, Eq. 2 is numerically integrated until the crack growth rate becomes unstable. The crack length at a given cycle is written as

$$
a_{N}=\int_{0}^{N} e^{C_{1}+C_{2} \log (K)} d N
$$

However, the load states can be discontinuous, and it is more appropriate to write it discretely as

$$
a_{N}=\sum_{N=0}^{N} e^{C_{1}+C_{2} \log (K)}
$$

When updating a previous model, the integration bounds are altered, and the summation is then written as 
$\left.a_{N+}=a_{N}+\sum_{N=N+1}^{N+} e^{C_{1}+C_{2} \log (}\right)$

However, it is most likely that the measured crack length at $(\mathrm{N})$ is a noisy measurement with some mean $(\mu)$ and variance $\left(\sigma^{2}\right)$, with a certain probability of detection/quantification. Considering the uncertainty, the distribution on crack length as a function of cycles becomes,

$p\left(a_{N+N}\right)=\sum_{N}^{N+N} e^{C_{1}+C_{2} \log (\quad)}+p\left(a_{N}\right)$

where $\mathrm{p}($.$) is the probability distribution.$

The uncertainty in the predicted crack length is due to the error associated with the calculation of $\Delta \mathrm{K}$ either analytically or numerically. Therefore, the measurement of $\Delta \mathrm{K}$ will have a mean value with some variance. This variance translates into the confidence in the prediction of the crack length.

Prior to integration, the non-constant coefficients, $C_{1}$ and $C_{2}$, must be determined. To calculate these coefficients, a least squares regression algorithm is used. The training data for the algorithm is heterogeneous in nature originating from multiple sources. Although only in-situ measured data is necessary to determine $C_{1}$ and $C_{2}$, introduction of additional data from previous experiments, expert knowledge, coefficients of Paris' Law, and advanced and multiscale models can drastically improve the results. The developed hybrid prognosis model can be applied at the first instance of crack initiation or at any measured point in time. The framework is numerically efficient and suitable for real time applications.

\section{A. Obtaining SIF for a given specimen:}

The only model parameter necessary as input for the proposed prognosis model is the stress intensity factor (SIF). An SIF mapping can be created for any specimen as a function of the crack tip location. Finite element simulations can be run for different crack tip locations, 
which will, in turn, serve as the training data. A regression model can then be used to map the data, allowing for evaluation of the SIF for any given crack tip location obtained from the experiments. Two different regression techniques, least absolute shrinkage and selection operator (LASSO) \& RVM are investigated in this study. LASSO is a deterministic regression model where as RVM is a fully probabilistic regression model. The difference between using both these methods is described in the subsequent sections. The results of the SIF mapping using both these methods are also provided in the subsequent sections. The model parameters used for regression are the crack growth rate and SIF. The regression is done on these parameters in the log-log scale to obtain the coefficients $\mathrm{C}_{1}$ and $\mathrm{C}_{2}$. Once the SIF is obtained for any given crack length from the mapping, and the coefficients obtained by fitting the data of previous crack growth rate and SIF in Eq. 2, the crack growth rate at any given cycle can be obtained. The future crack length for any given number of cycles then can be obtained using Eq. 6 .

\section{B. LASSO}

Tibshirani [34] proposed the LASSO technique to improve the accuracy of regression and eliminate the outliers that contribute to the error. Brief details of this method are provided as reference. Let the data points be $\left(x^{i}, y_{i}\right), i=1,2, \ldots, N$, where $x^{i}=\left(x_{i l}, \ldots, x_{i p}\right)$ are the predictor variables and $y_{i}$ are the responses.

Let $\beta=\left(\beta_{1}, \ldots, \beta_{p}\right)^{T}$, the LASSO estimate $(\alpha, \beta)$ is defined by

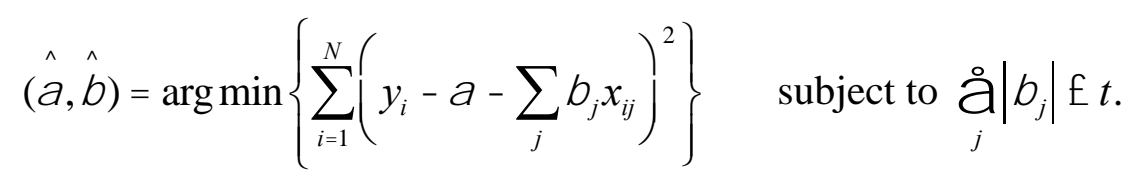

Here $t \geq 0$ is a tuning parameter. The solution to the above equation is a quadratic programming problem with linear inequality constraints. The parameter $t$ controls the amount of shrinkage that is applied to the estimates. Let $\beta_{j}^{o}$ be the full least squares estimates and let $t_{0}=\Sigma \mid$ 
$\beta_{j}^{o} \mid$. Values of $t<t_{0}$ will cause shrinkage of the solutions towards zero, and some coefficients may be exactly equal to zero. This leads to the sparseness in the solution, eliminating the outliers in the process. The accuracy of the fit depends on the type of kernel function used. Kernel functions are continuous, symmetric, and generally have a positive (semi)-definite Gram matrix. Positive semi-definite kernels satisfy the Mercer's theorem, which ensures that the kernels have no non-negative eigenvalues. A positive definite kernel means that the optimization problem is convex and thus ensures a unique solution. The choice of kernel depends on the data being modeled. A polynomial kernel can be used to model feature conjugations up to the order of the chosen polynomial. A radial basis function kernel is used to model circles or hyperspheres.

\section{RVM}

Tipping [35] introduced a technique called the RVM, which is an improvement over the support vector machine (SVM), and is fully probabilistic. In supervised learning, a set of examples of input vectors $\left\{x_{n}, n=1, \ldots, N\right\}$ along with corresponding targets $\left\{t_{n}, n=1, \ldots, N\right\}$ are given. The aim is to set up a model of the dependency of the targets on the inputs with the objective of making accurate predictions of $t$ for previously unseen values of $\mathrm{x}$. Generally, predictions are based upon some function $\mathrm{y}(\mathrm{x})$ defined over the input space, and 'learning' is the process of inferring this function. A function $\mathrm{y}(\mathrm{x})$ can be written in the form:

$$
y(x ; w)={ }_{i=1}^{M} w_{i} \quad(x)=w^{T}(x)
$$

where the output is a linearly-weighted sum of $M$ nonlinear and fixed basis functions

$$
(x)=\left({ }_{1}(x),{ }_{2}(x), \ldots, \quad{ }_{M}(x)\right)^{T}, w=\left(w_{1}, w_{2}, \ldots, w_{M}\right)^{T}
$$


The adjustable parameters $\mathrm{w}=\left(w_{1}, w_{2}, \ldots, w_{M}\right)^{T}$ appear linearly, and the objective is to estimate the optimal values for these parameters.

The drawbacks of the SVM are that several basis functions are used and their numbers increase with the training data. Also, the predictions are not probabilistic and the kernel function must satisfy the Mercer's condition, which requires it to be continuous symmetric. The RVM approach is a Bayesian treatment of the SVM and does not have any of the above limitations. It is a fully probabilistic framework where a prior over the weights is governed by a set of hyperparameters, one associated with each weight, and the most probable values are iteratively estimated from the data. The posterior distributions of many of the weights are peaked around zero achieving sparsity. We consider similar type of functions as those implemented in SVM, $y(x ; w)={ }_{i=1}^{N} w_{i} K\left(x, x_{i}\right)+w_{0}$

where, $\mathrm{K}\left(\mathrm{x}, \mathrm{x}_{i}\right)$ is the kernel function. However, an important advantage of RVM is the fact that considerably fewer number of kernel functions are used. In addition to offering good accuracy, the predictors are sparse and contain few non-zero $w_{i}$ parameters since the majority of the parameters are set to zero during the learning process; moreover, only those that are relevant are used for optimal predictions.

Given a data set of input-target pairs $\left\{x_{n}, t_{n}, n=1, \ldots, N\right\}$ and considering scalar-valued target functions from the standard probabilistic formulation alone, we can assume that the targets are samples from the model having additive noise:

$t_{n}=y\left(x_{n} ; w\right)+{ }_{n}$

where the noise is assumed to be a mean-zero Gaussian with a variance $\sigma^{2}$. Thus, $p\left(t_{n} \mid x\right)=\left(t_{n} \mid y\left(x_{n}\right), \quad{ }^{2}\right)$ 
is a Gaussian distribution over $t_{n}$ with a mean $y\left(x_{n}\right)$ and variance $\sigma^{2}$. Due to the assumption of the independence of $t_{n}$, the likelihood of the complete data set can be written as,

$p\left(t \mid w,{ }^{2}\right)=\left(2^{2}\right)^{N / 2} \exp \left\{\frac{1}{2^{2}}\|t \quad w\|^{2}\right\}$

where, $\mathrm{t}=\left(\begin{array}{lll}t_{1} & \ldots & t_{N}\end{array}\right)^{\mathrm{T}}, \mathrm{w}=\left(\begin{array}{lll}w_{1} & \ldots & w_{N}\end{array}\right)^{\mathrm{T}}$, and is the $N \times(N+1)$ design matrix with $=\left[\left(x_{1}\right),\left(x_{2}\right), \ldots,\left(x_{N}\right)\right]^{T}$

As there is a hyperparameter associated with each training point in the data, the maximum likelihood estimation of $w$ and $\sigma^{2}$ is expected to lead to overfitting. To avoid this, a common approach is to impose an additional constraint in the form of a penalty term. Here, the parameters are constrained because an explicit prior probability distribution is defined over them. We choose a zero-mean Gaussian prior distribution over $w$ $p(w \mid)={ }_{i=0}^{N} \quad\left(w_{i} \mid 0,{ }_{i}^{1}\right)$

where $\alpha$ is a vector of $\mathrm{N}+1$ hyperparameters. Then, we define a prior over $\alpha$ and the noise variance, $\sigma^{2}$. These parameters are scale parameters, and hence the prior is defined by a Gamma distribution,

$$
\begin{aligned}
& p(\quad)={ }_{i=0}^{N} \operatorname{Gamma}\left({ }_{i} \mid a, b\right), \\
& p(\quad)={ }_{i=0}^{N} \operatorname{Gamma}(\mid c, d),
\end{aligned}
$$

with $={ }^{2}$, and

$\operatorname{Gamma}(\mid a, b)=(a){ }^{1} b^{a}{ }^{a}{ }^{1} e^{b}$,

where, $\quad(a)={ }_{0} t^{a}{ }^{1} e^{t} d t$ is the "gamma function". 
The parameters $\alpha$ and $\beta$ are set to the hyper-parameter posterior mode as:

$p\left(t_{*} \mid t, \quad{ }_{M P}, \quad \underset{M P}{2}\right)=\quad p\left(t_{*} \mid w, \quad \stackrel{2}{M P}\right) p(w \mid t, \quad \underset{M P}{ }, \quad \stackrel{2}{M P}) d w$

where ${ }_{M P},{ }_{M P}^{2}$ are the most probable values. Both the terms in the integrand are Gaussians, hence giving:

$p\left(t_{*} \mid t, \quad \begin{array}{ll}2 & 2 \\ M P\end{array}\right)=\left(t_{*} \mid y_{*}, \quad \stackrel{2}{*}\right)$

with,

$$
\begin{aligned}
y_{*} & ={ }^{T}\left(x_{*}\right) \\
{ }_{*}^{2} & ={ }_{M P}^{2}+\left(x_{*}\right)^{T} \quad\left(x_{*}\right)
\end{aligned}
$$

where $y_{*}$ and $\quad{ }_{*}^{2}$ are the mean and variance of the predicted value.

\section{Validation \& Results}

\section{A. Constant amplitude loading}

The efficiency of the hybrid prognosis model is illustrated using a compact tension (CT) test article with available test data. $\mathrm{Wu}$ and $\mathrm{Ni}[36]$ conducted 30 constant amplitude fatigue tests on the CT samples to generate a statistically large dataset and concluded that the results were log-normally distributed. Compact tension specimens, $50 \mathrm{~mm}$ wide and $12 \mathrm{~mm}$ thick, were fabricated from 2024-T351 aluminum alloy according to the ASTM standard E647-93 [37]. The specimens were pre-cracked up to $15 \mathrm{~mm}$ and extended to $18 \mathrm{~mm}$ of crack length. A constant amplitude load, with maximum amplitude of $4.5 \mathrm{kN}$ and minimum amplitude of $0.9 \mathrm{kN}$, was applied during both the pre-cracking and fatigue tests. The crack lengths were measured using images taken by a microscope, until the specimen fractured. The constant amplitude-loading envelope is shown in Fig. 4. 


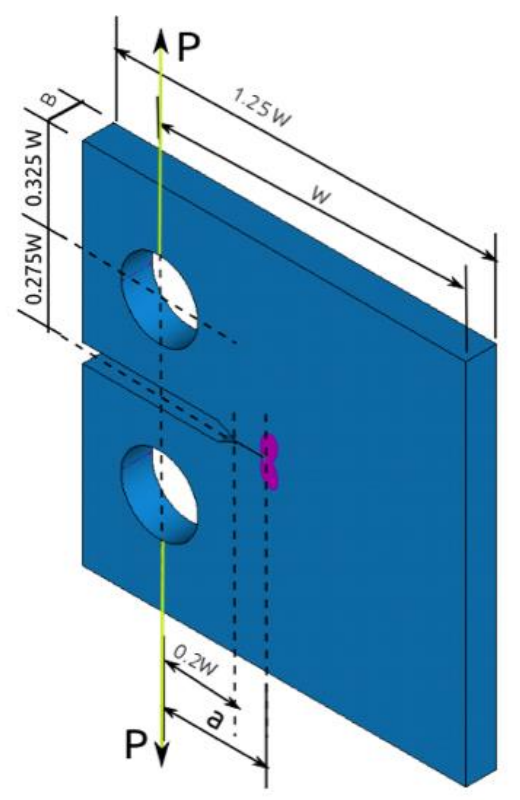

Fig. 3 The CT specimen is subjected to constant and random amplitude loading while the crack length is monitoring as a function of cycles.

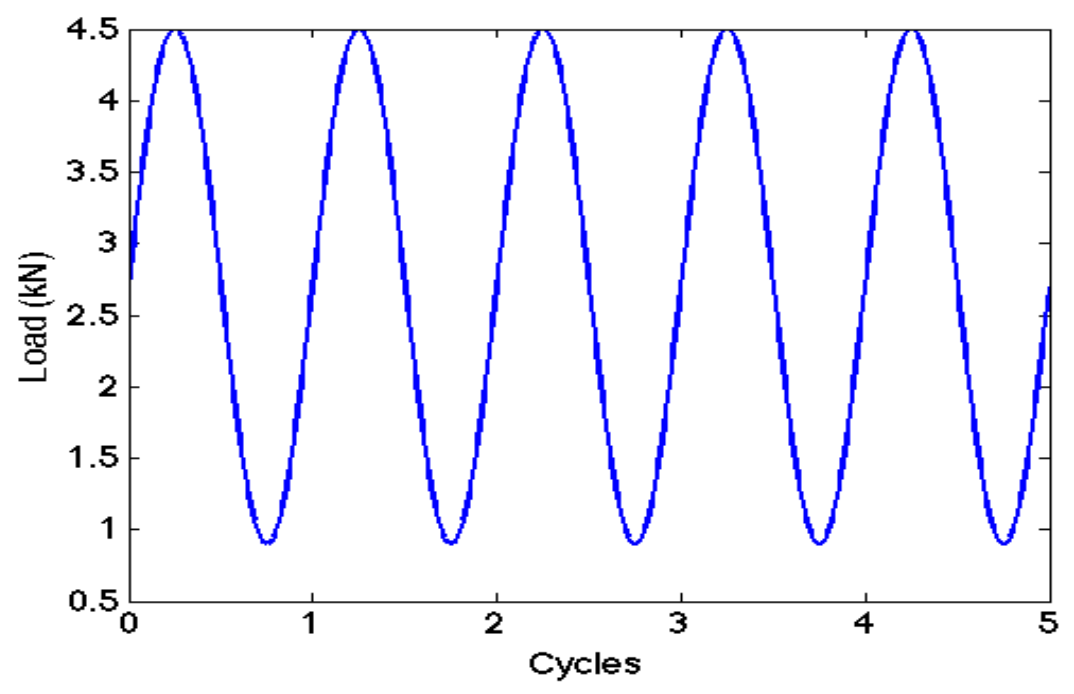

Fig 4. Constant amplitude loading envelope

An analytical expression for the $\operatorname{SIF}(\mathrm{K})$, as a function of the crack length $(a)$, geometry and load for a CT specimen was used to solve the differential equation shown in Eq. 2. This is shown in Eq. 20 and the variables are defined in Fig. 3. 


$$
\begin{aligned}
K_{\max } & =\frac{P_{\max }}{B} \sqrt{\frac{\pi}{W}}\left[16.7\left(\frac{a}{W}\right)^{1 / 2}-104.7\left(\frac{a}{W}\right)^{3 / 2}+369.9\left(\frac{a}{W}\right)^{5 / 2}-573.8\left(\frac{a}{W}\right)^{7 / 2}+360.5\left(\frac{a}{W}\right)^{9 / 2}\right] \\
K_{\min } & =\frac{P_{\min }}{B} \sqrt{\frac{\pi}{W}}\left[16.7\left(\frac{a}{W}\right)^{1 / 2}-104.7\left(\frac{a}{W}\right)^{3 / 2}+369.9\left(\frac{a}{W}\right)^{5 / 2}-573.8\left(\frac{a}{W}\right)^{7 / 2}+360.5\left(\frac{a}{W}\right)^{9 / 2}\right] \\
K & =K_{\max } K_{\min }
\end{aligned}
$$

where, $P_{\max }$ is the maximum amplitude and $P_{\min }$ is the minimum amplitude of the cyclic loading. To start the prediction at a given cycle, the non-constant coefficients must be first determined. This was achieved through linear fitting of all acquired data points (i.e., all known $\frac{d a}{d N}$ and $K$ ) with two additional training data points. In order for the data to regress, they must be transformed from crack length versus cycles to crack growth rate versus SIF. Numerical differentiation and Eq. 4 were used to accomplish this. The training data was derived from Paris' Law coefficients using the average response of a 30 specimen dataset. The Paris' Law coefficients were used to evaluate the crack growth rate and the SIF at the approximate start and end of the Stage II crack growth, points that are far away from the measured dataset. As the crack length increased and more data were available, the weight of the training data is reduced, relying primarily on the measured data. Once the coefficients are evaluated, the RUL can be estimated based on the number of cycles required for the crack to reach a critical length. Considering a very small number of cycles $(N)$, the crack length at $(N+N)$ cycles can be obtained as,

$$
a_{N+\Delta N}=a_{N}+\frac{d a}{d N} \Delta N
$$

where $a_{N}$ is the crack length after $N$ cycles. 

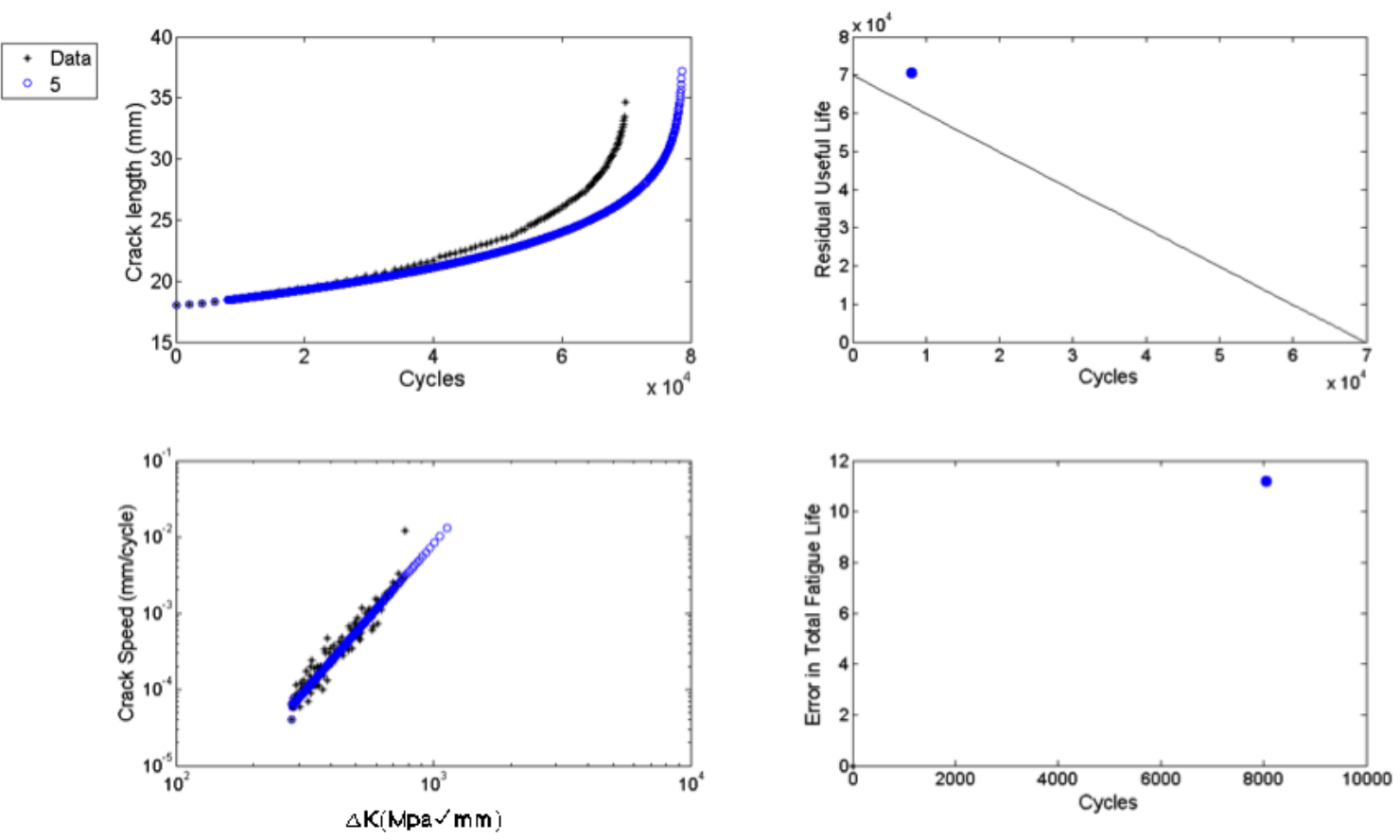

Fig. 5 Initial prediction starting at 8000 cycles

The methodology described here was applied to several starting points to demonstrate the convergence of RUL as more data became available. A random dataset was chosen [36] to validate the hybrid prognosis framework. The experimental crack length dataset and initial prediction as well as the data transformed into prediction space are shown in Fig. 5. In Fig. 5, the first subplot shows the variation of crack length with the number of cycles. The maximum crack length before fracture was $35 \mathrm{~mm}$. The second subplot shows the RUL estimation at different stages of the crack growth regime. The dark line shows the actual RUL of the specimen. If the estimated RUL falls above the line, it indicates over-prediction of the RUL, and if it falls under the line, it indicates under-prediction. The third subplot shows the relationship between $K$ and the crack growth rate. Although this plot is nonlinear, it can be assumed linear for every step. As this hybrid model is updated in steps, as more data is available, the coefficients $\mathrm{C} 1$ and $\mathrm{C} 2$ are updated. If the interval between two data points is small, then the behavior can be assumed to be 
linear in that interval. The fourth subplot shows the error in estimation of the RUL at different stages of the crack growth regime. The error is defined by (RUL RUL predicted)/RUL. The regression is performed on the first five data points plus two additional points mentioned above. The two additional data points would be the first and last data points corresponding to the $\mathrm{x}$-axis in the third subplot. The legend on the top left corner of the figure shows the index of the data point for which the prediction is made. The cumulative results of several predictions superimposed on Fig. 5 are shown in Fig. 6.
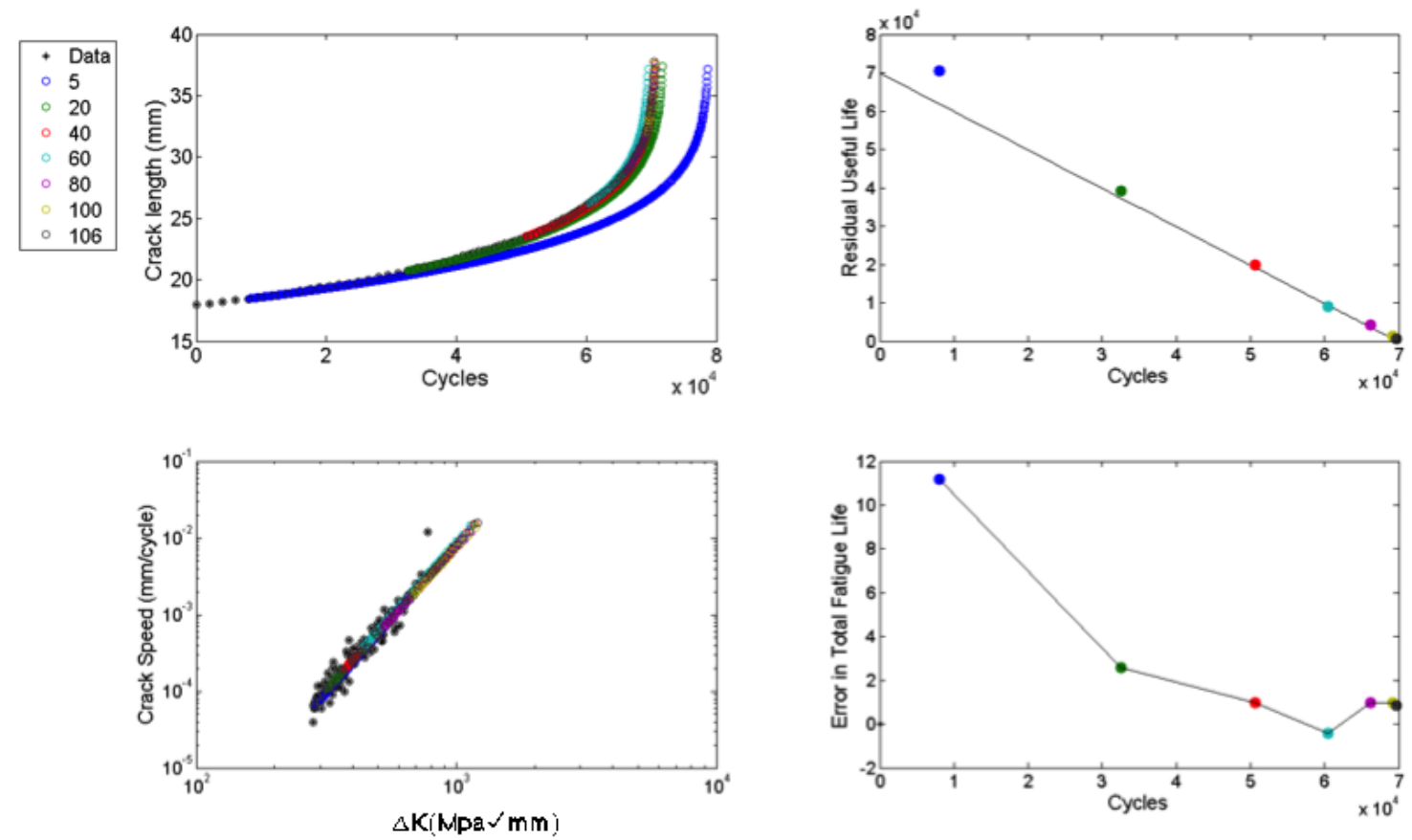

Fig. 6 Multiple predictions made using the hybrid framework simulating a real-time experiment

The prior results utilize all available data to determine the non-constant coefficients of Eq. 2. However, by reducing the amount of data used for regression, the algorithm is more suited for adapting to small changes in crack growth rates. To illustrate this, only the last five available data points were used for regressing Eq. 2. The predictions starting at the fifth available data point and considering only the previous five points for regression are shown in Fig. 7. It is 
to be noted that the regression curves in Fig. 6,7,14,15,16a-6c are not drawn for the whole abscissa scale for presentation purposes. If more regression curves are plotted, they overlap with each other and will be difficult to distinguish. Hence, a representative sample of the regression curves which show the maximum and minimum deviation from the original crack length data were plotted.
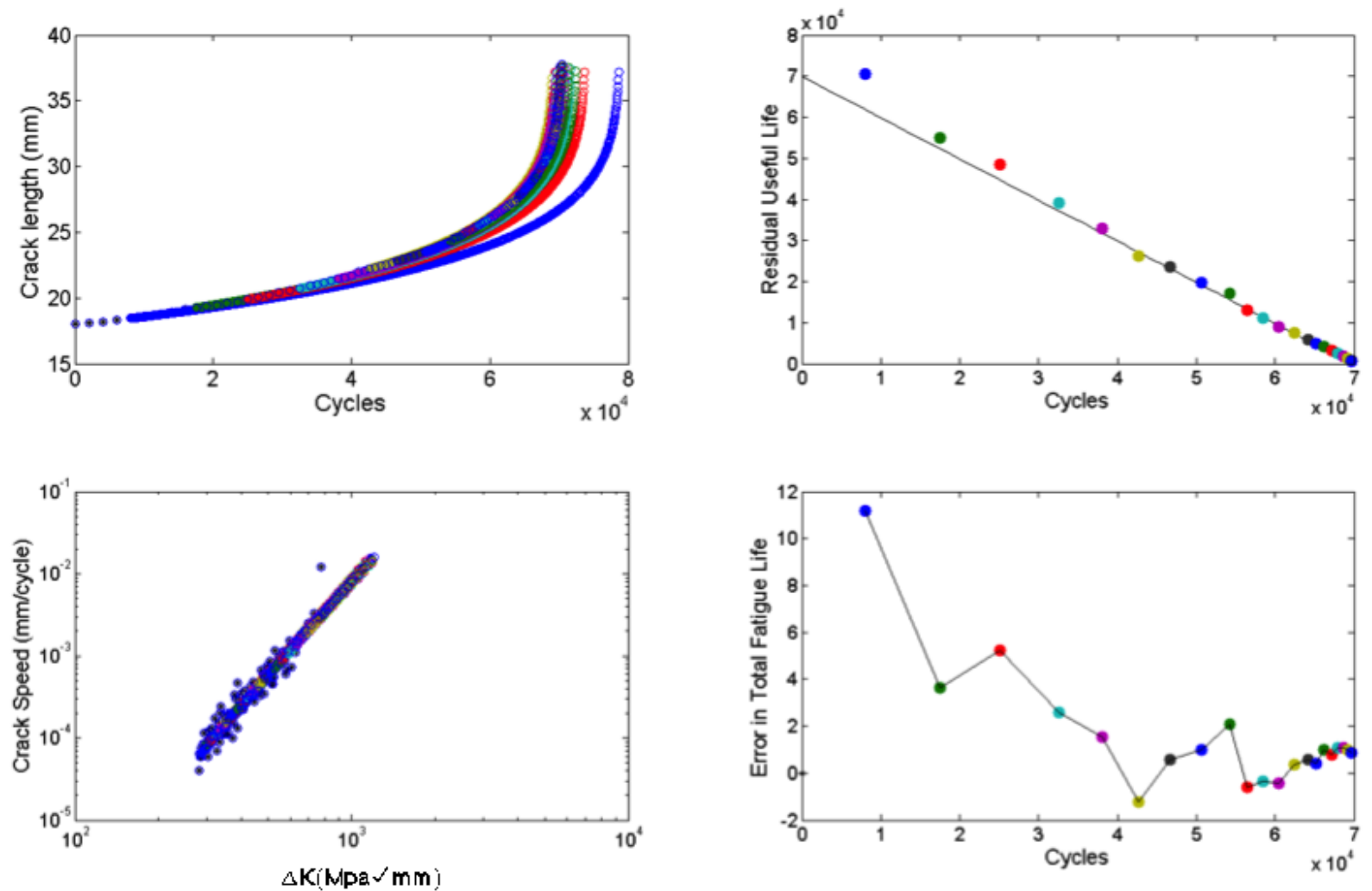

Fig. 7 Multiple predictions are made using previous five data points at every iteration 
The results of Fig. 7 show the capability of the hybrid model to predict the RUL with an error of less than $10 \%$ during the initial crack growth regime and $2 \%$ towards the end.

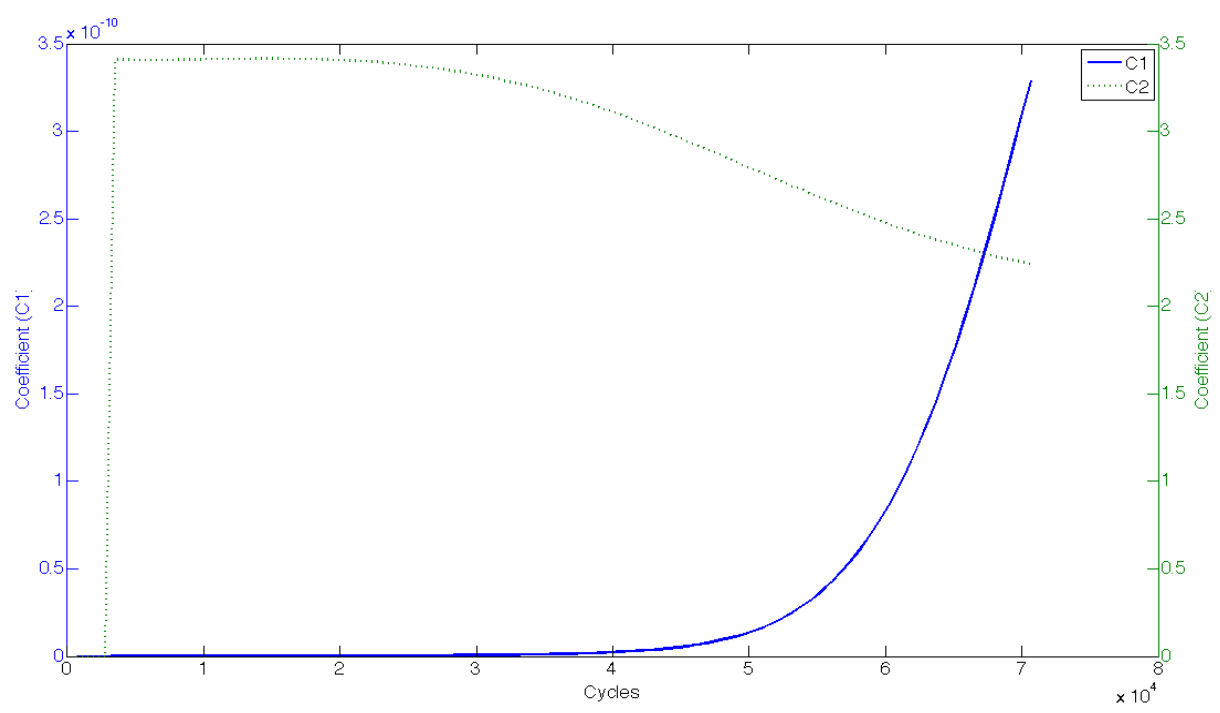

Fig. 8 Variation of the coefficients $\left(C_{1} \& C_{2}\right)$ with number of cycles for constant amplitude loading

The variation of coefficients $C_{1} \& C_{2}$ with the number of cycles is shown in Fig. 8. The coefficients start from a very low value and as more data is obtained, they adapt to give better estimates.

\section{B. Random amplitude loading}

$\mathrm{Wu}$ and $\mathrm{Ni}$ [36] published a dataset for the random loading of specimens similar to those used in the constant amplitude-loading test. A band-limited $(5-15 \mathrm{~Hz})$ and uniformly distributed power spectrum density function was used to generate random signals with a mean value of 5 $\mathrm{kN}$, a random amplitude of mean of $1.118 \mathrm{kN}$, and a standard deviation of $0.552 \mathrm{kN}$. The profile of the loading envelope is shown in Fig. 9. 
The SIF calculation and training methodology was identical to that of the constant loading. However, the training data was modified based on the mean values of the random dataset. The results for multiple predictions are shown in Fig. 10.

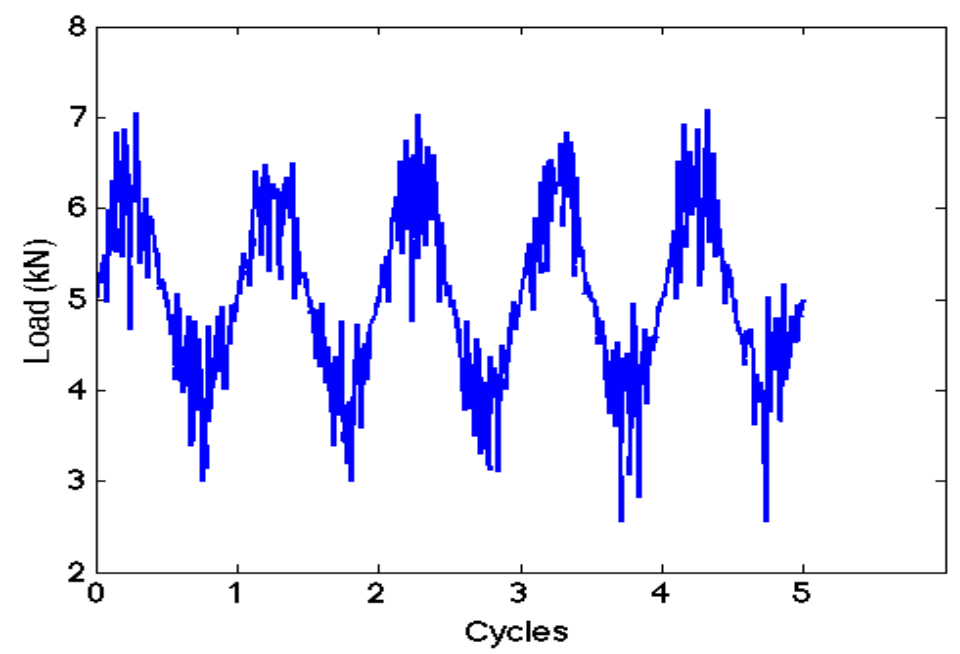

Fig 9. Profile of the random loading envelope
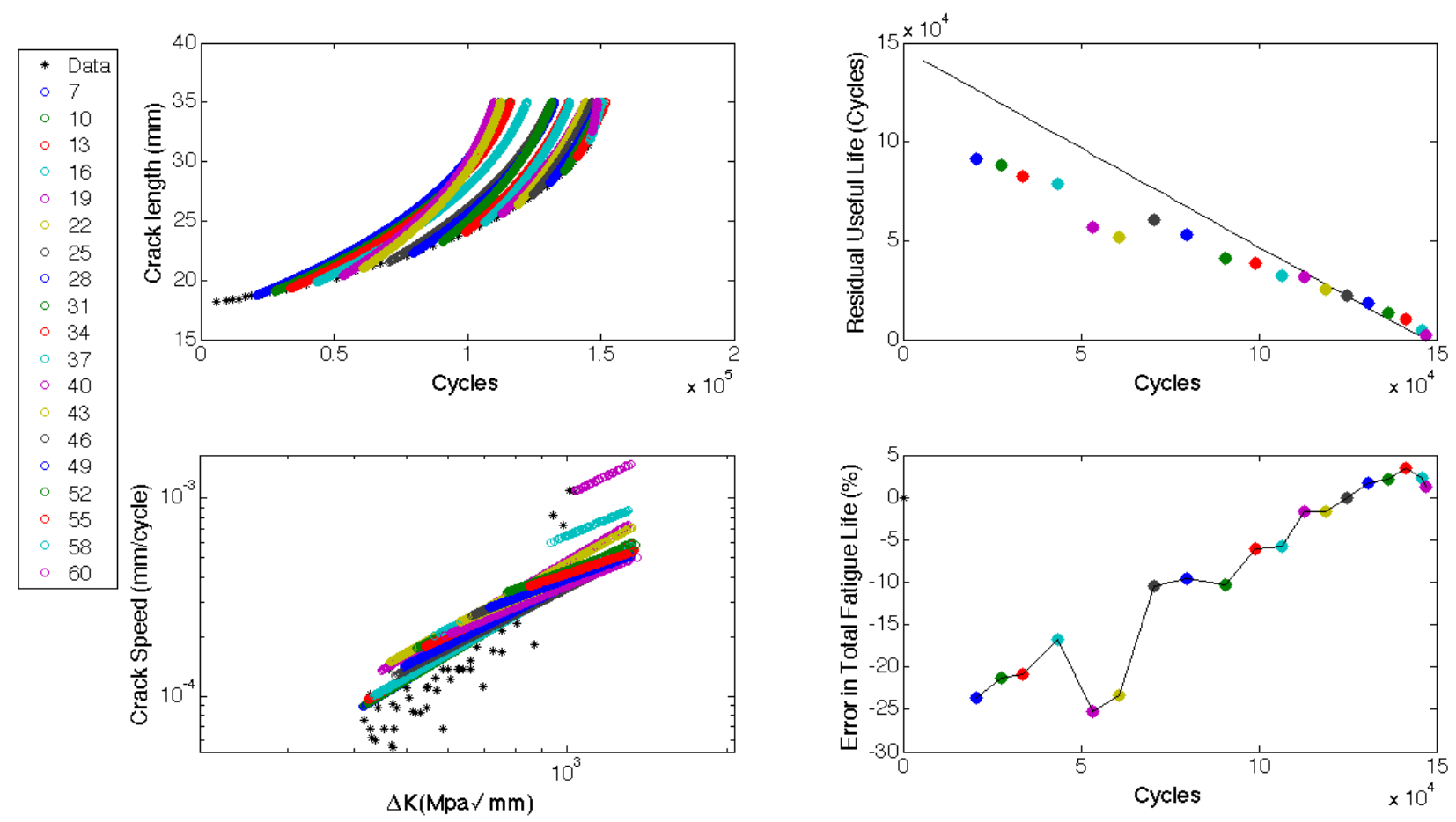

Fig. 10 Multiple predictions are made under random loading using only previous five data points at every iteration. 
The results in Fig. 10 show the capability of the hybrid model to predict RUL under random loading conditions. The model is able to predict the RUL with an error of less than 5\% as more training data becomes available.

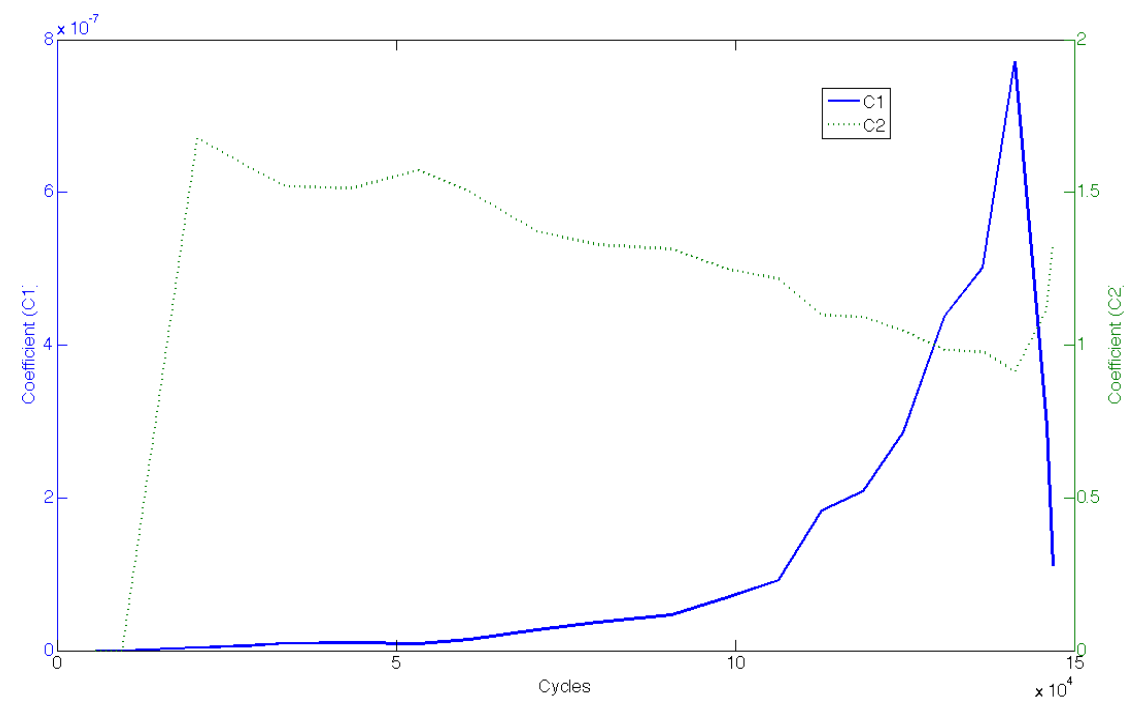

Fig. 11 Variation of the model parameters $\left(C_{1} \& C_{2}\right)$ with number of cycles for random loading

The variation of coefficients $\mathrm{C}_{1} \& \mathrm{C}_{2}$ is shown in Figure 11. The coefficients start from a very low value and adapt themselves to give accurate estimates of RUL as more data is obtained.

\section{Overload}

McMaster and Smith [38] presented a dataset for a center-cracked specimen made from an Al 2024-T351 alloy under an overload. The specimen was $100 \mathrm{~mm}$ wide, $250 \mathrm{~mm}$ long and $14 \mathrm{~mm}$ thick. The overload test consisted of three overload excursions applied at crack length intervals of $2 a / W=0.4,0.5,0.6$. A $4 \mathrm{~mm}$ hole was made at the center of the specimen, followed by electro-discharge machining of a starter notch of length $2 \mathrm{~mm}$ and height $0.2 \mathrm{~mm}$. The geometry of the test article is shown in Fig. 12. 


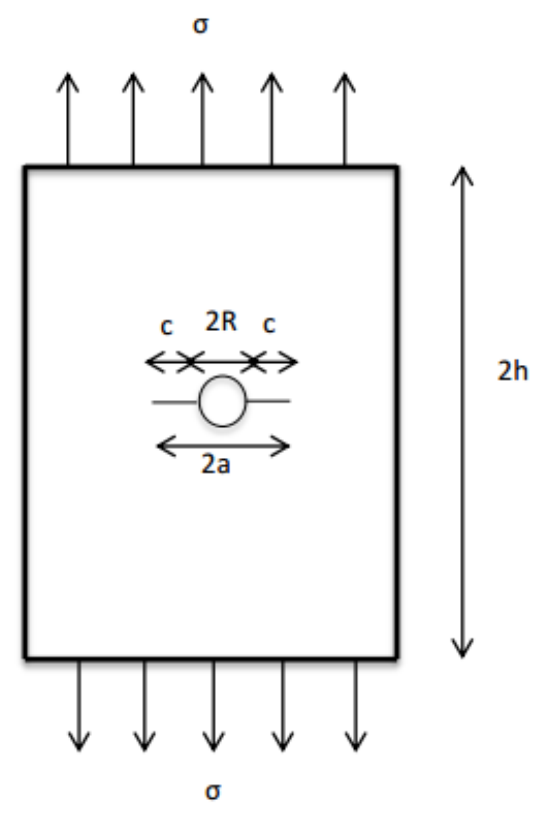

Fig. 12 Plate with a central hole [40] under constant amplitude loading with overload at specific intervals The SIF for the center-cracked plate [39] is calculated as,

$$
\begin{aligned}
& K=F \quad \sqrt{a} \\
& \text { where, } \\
& F=\text { * } \\
& =\frac{a}{W},=\frac{a}{2} a,=\frac{a}{R},=\frac{R}{W},=\frac{}{1} \\
& =\frac{\left[\sqrt{\left.\left.\frac{1}{-(\tan +g \sin 2}\right)\left(1+\frac{{ }^{2}\left(2^{2}\right)}{1+}\right)\right] \sqrt{1+2 g}}\right.}{1} \\
& g=0.13\left(\frac{2}{\arctan }\right)^{2},=\frac{2}{\arctan }(0.6 \sqrt[3]{ }) \\
& =\left(3^{\frac{2 P}{3}} 2 \sqrt{ }^{P}\right), P=\frac{\log \left(^{3 / 2}\right)}{\log { }^{*}}
\end{aligned}
$$

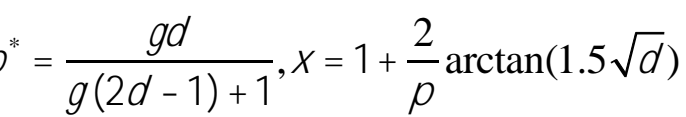

where $a$ is the crack length, $F$ is the geometric factor, and $\sigma$ is the magnitude of applied loading. 
To capture the crack closure phenomenon associated with overloads, the algorithm for determining the non-constant coefficients was modified. Typical overload behavior in the log-log plot of crack growth rate versus SIF [39] is illustrated in Fig. 13. A linear growth rate is observed in stage II; however, once the specimen has been overloaded, the crack closure phenomenon [40] reduces the growth rate slope significantly. This new behavior continues until it reaches the original linear response. However, since the hybrid prognosis model adapts to new data, an initial linear model is more than adequate to yield good predictions. For this sample, training data used to calculate the slopes of the overload region were averaged from the experimental data.

A brief overview of the algorithm is provided for clarity. Initially, as there is no overload, the algorithm predicts the RUL with the same method as for random loading. Once there is an overload, the crack growth rate decreases significantly. In our model, we assume that after an overload, the crack growth rate is reduced to a fixed value and the exponential coefficient, $\mathrm{C} 2$, is set to a high value, and estimates of these parameters are taken from training data. Once an overload is detected, the algorithm starts to fit a new line from the data point at which the overload occurs. The fitting of this new line will continue until the slope of the line obtained using the new data point matches with the slope of the original fit before the overload. This procedure will be repeated for different overloads to estimate the RUL. 


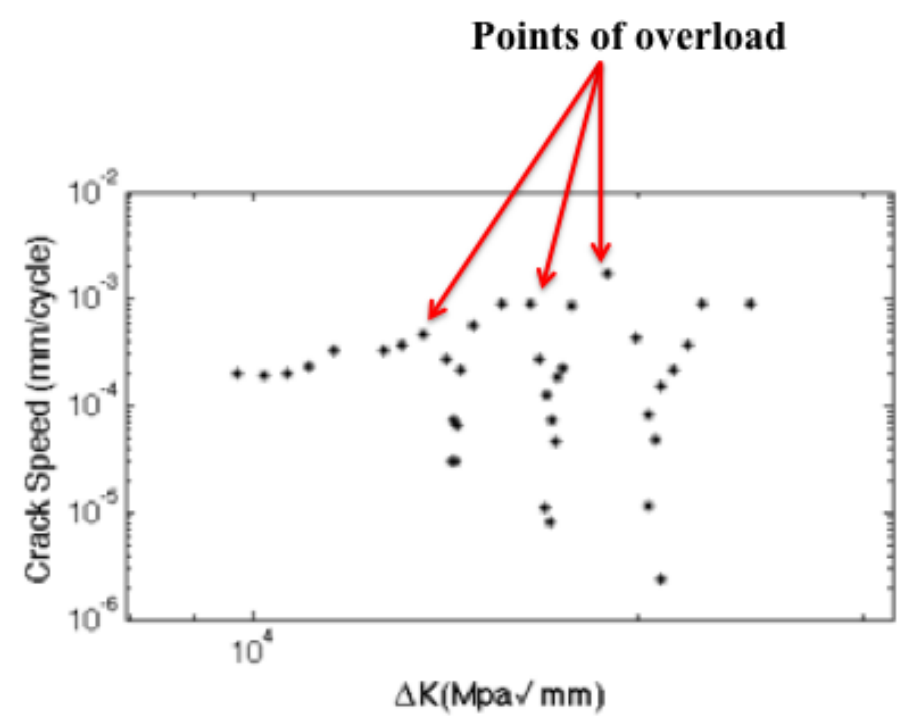

Fig. 13 The effect of overload on the crack growth rate and SIF causes a discontinuity in the curve resulting in crack closure.

In order to consider overloads in RUL, the times at which the overload excursions occur must be either assumed or modeled. To make the prediction, the cycles at which the experimental overloads occurred were assumed to be known, similar to an "oracle" approach. Here the post overload crack growth rate was set to $5 \mathrm{e}-5 \mathrm{~m} / \mathrm{s}$ and the $\mathrm{C} 2$ coefficient was set to 29. These results are shown in Fig. 14.

However, if the number of overloads and the times when they occur are unknown (as is the case for most problems), the prediction results can vary and are strongly dependent on load occurrence. For example, the same specimen was simulated with four overloads at random cycles. This is shown in Fig. 15. The results show prediction capabilities within 5\% for randomized future loading. 

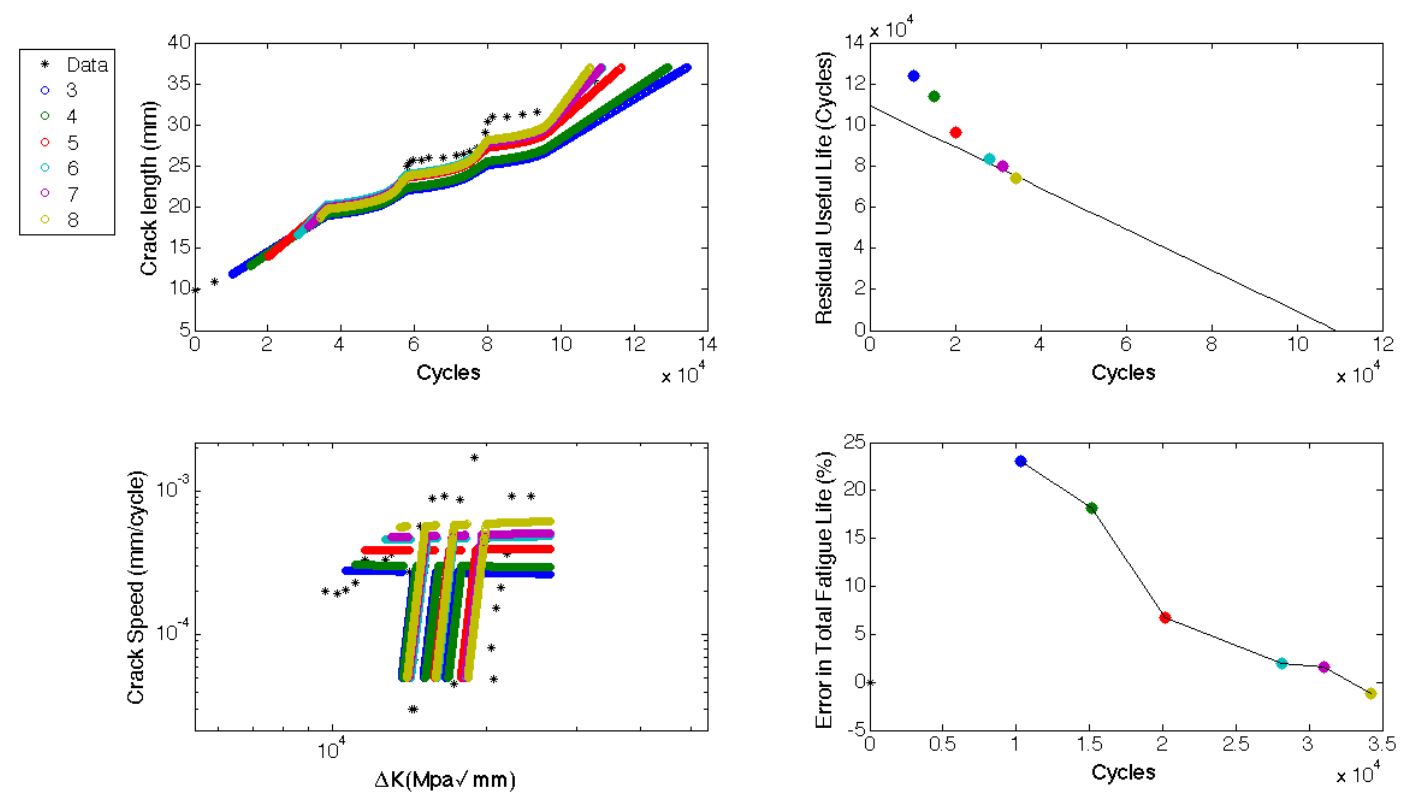

Fig. 14 Multiple predictions are made with known overload data. The results predict very well with-in an error of $5 \%$ as the overload point is known
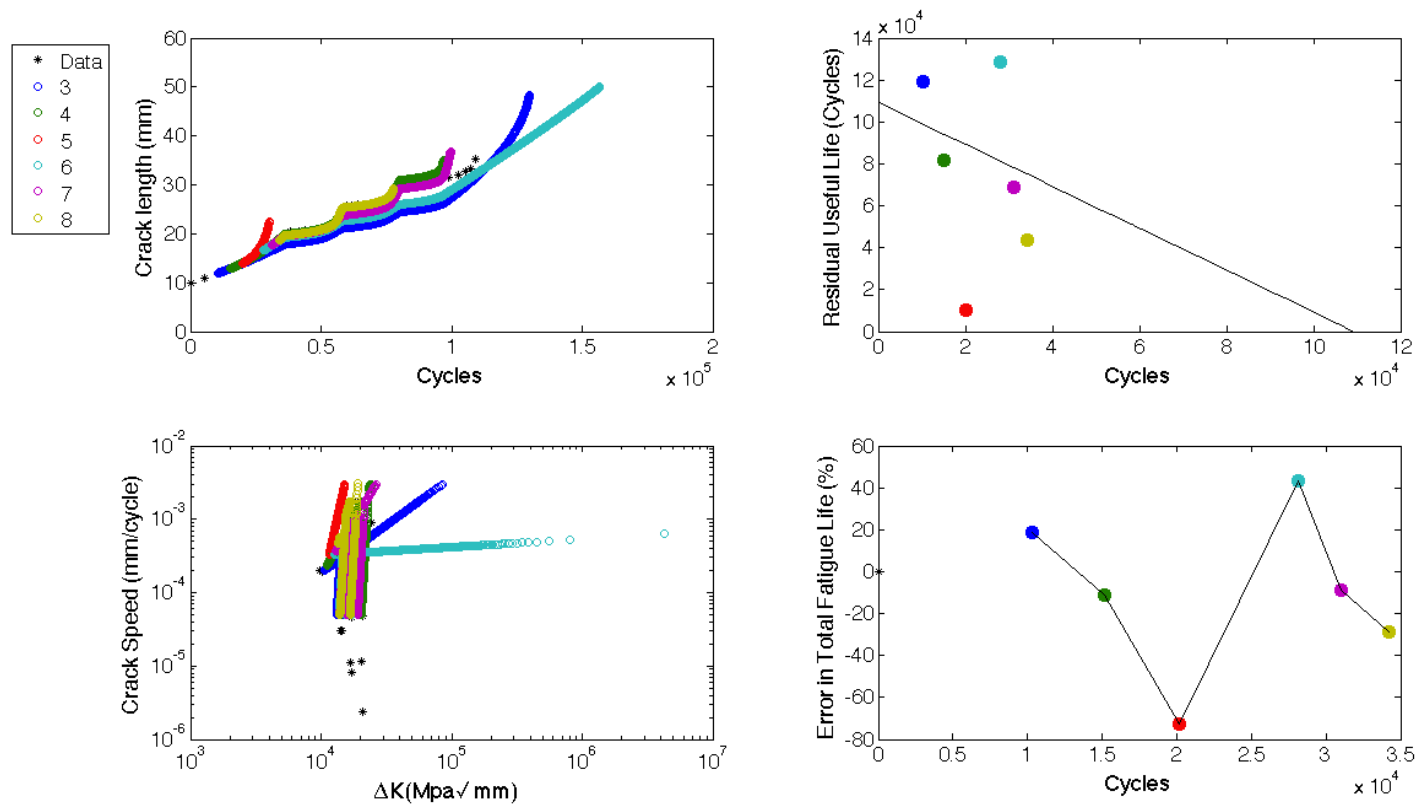

Fig. 15 Multiple predictions made using unknown overload data.

Next, the number of overloads was assumed randomly and the algorithm was run for different cases. Fig. 16a-16c shows the prediction for different number of assumed overloads. 

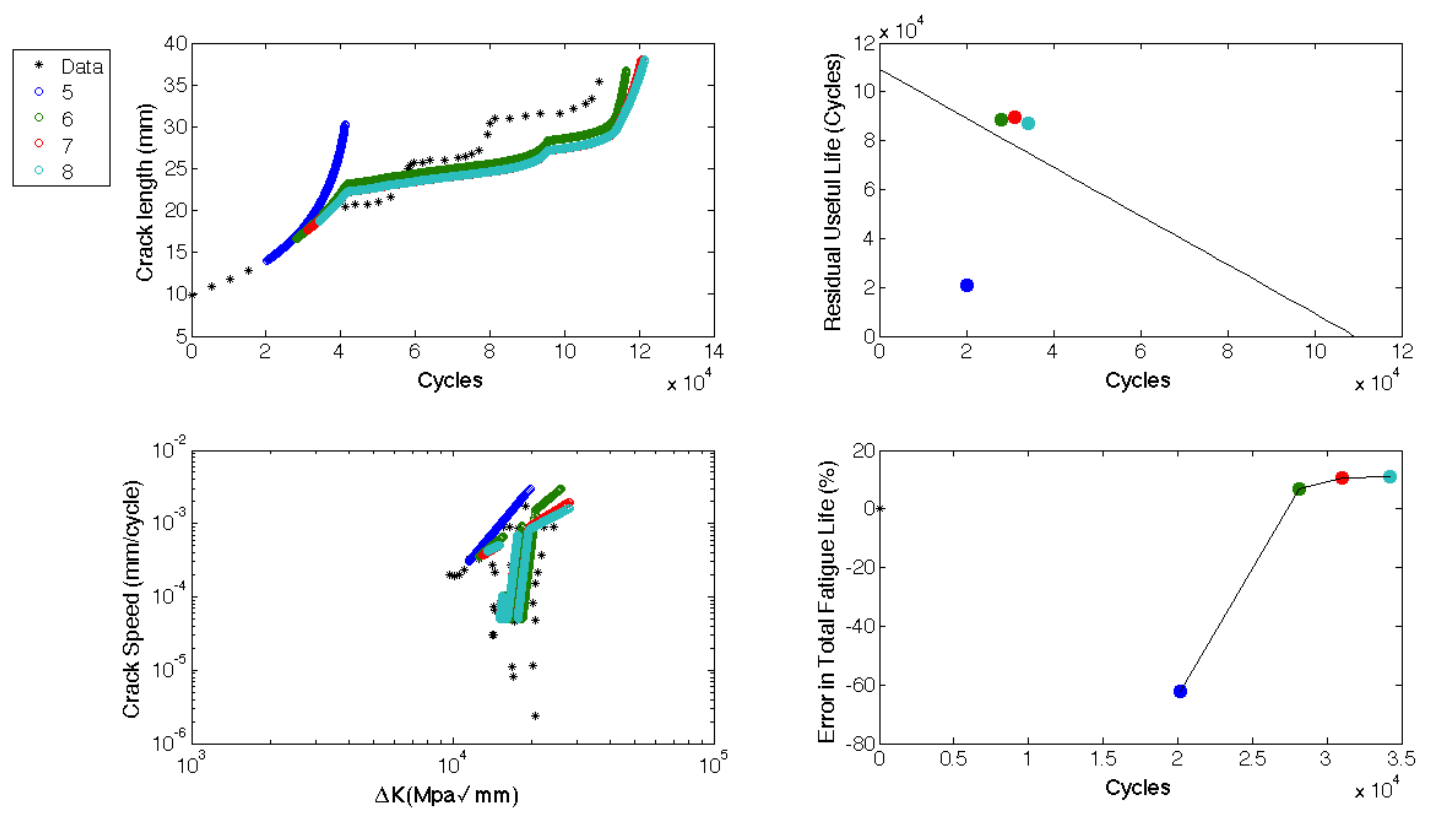

Fig 16a. Predictions with number of assumed overloads as "2"
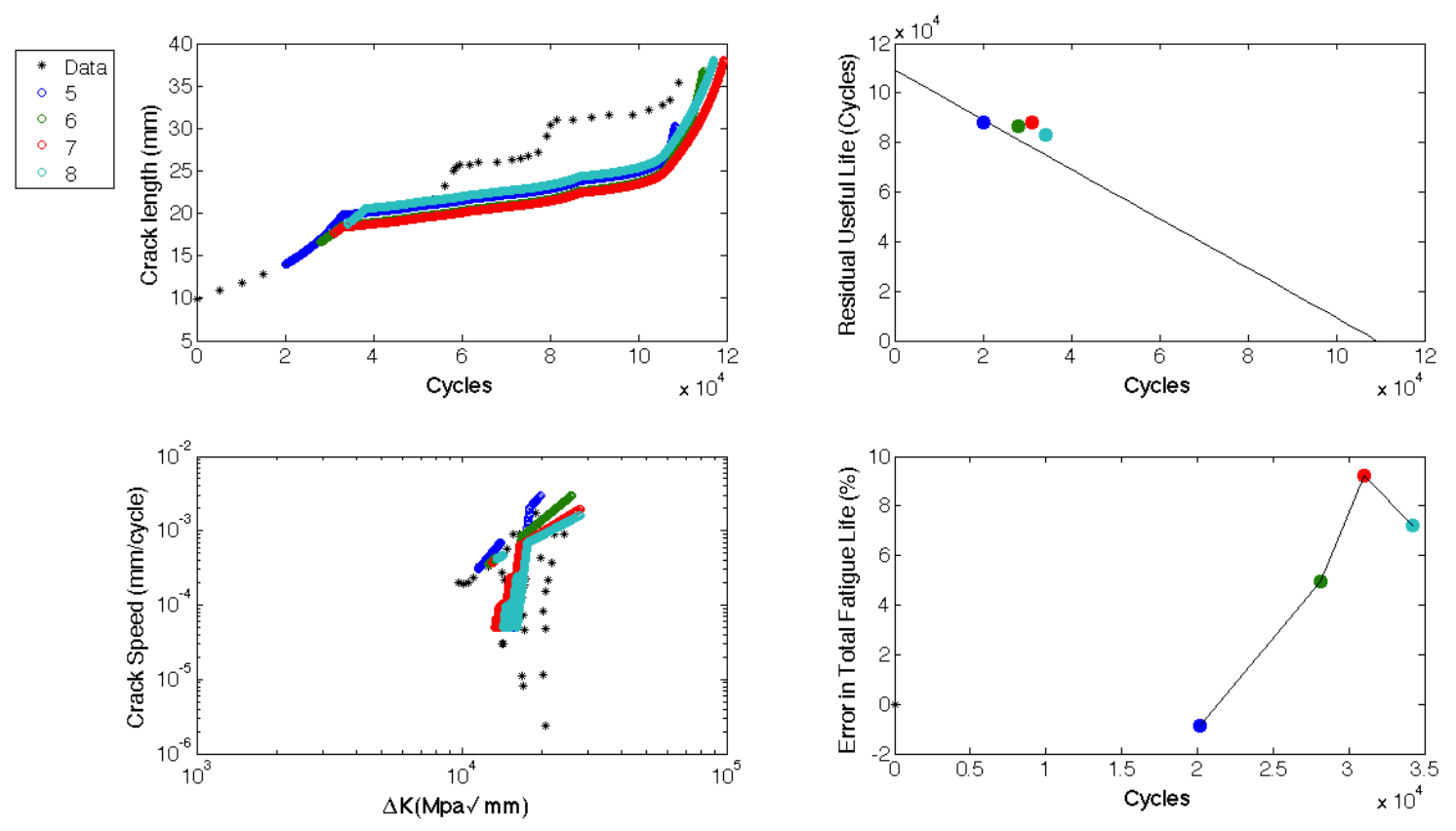

Fig 16b. Predictions with number of assumed overloads as " 4 " 

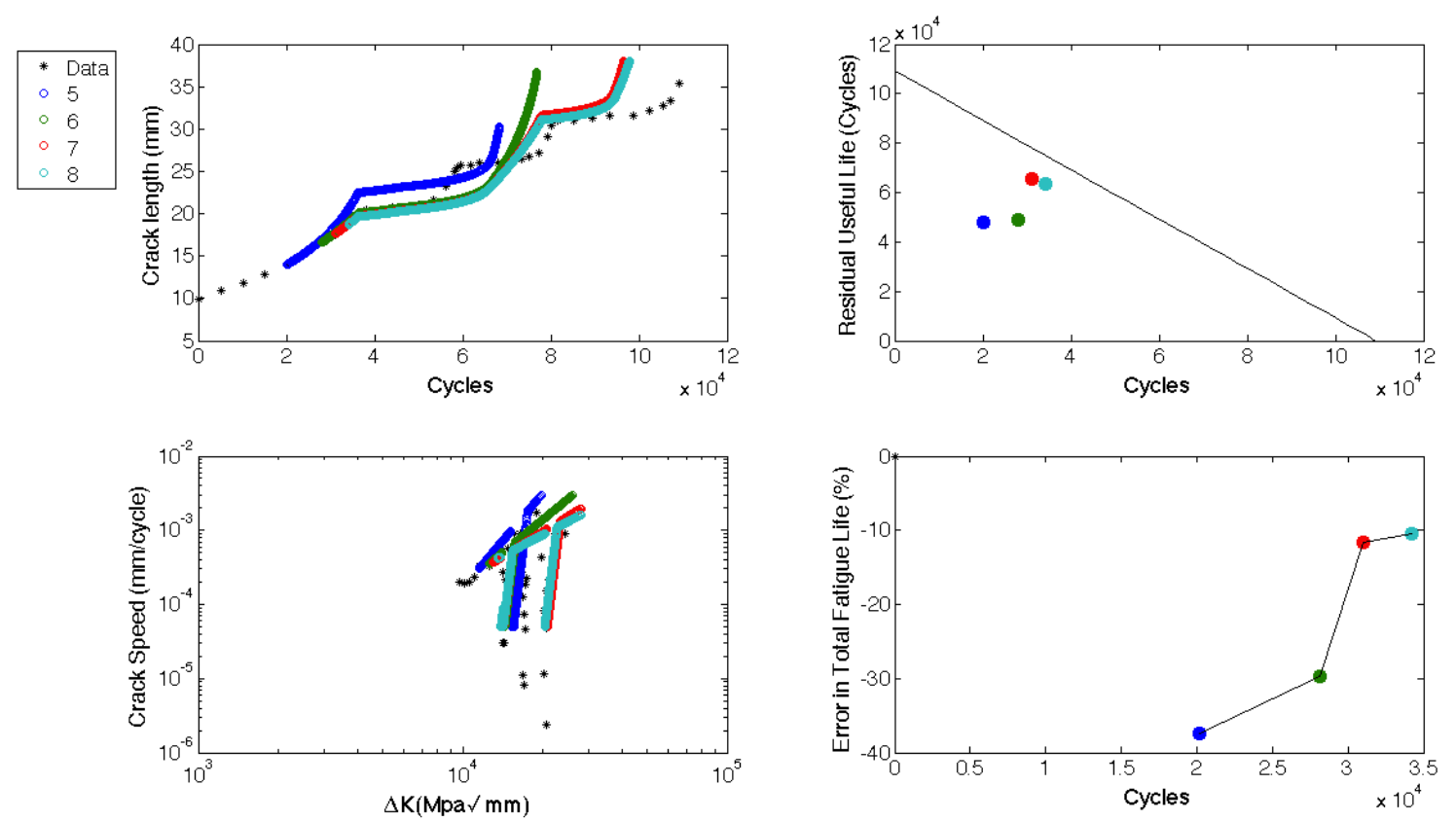

Fig 16c. Predictions with number of assumed overloads as "5"

From the Fig. 16a-16c, we can see that even though the number of overloads and the times at which they occur are unknown, the algorithm adapts to the changes in the behavior and accurately predicts the RUL with an error of less than $\pm 10 \%$.

\section{Experimental validation}

For the validation of the method on a real-time specimen, an Al 2024-T351 lug joint subjected to fatigue loading was instrumented and interrogated. The dimensions of the lug joint are given in Fig. 17. 


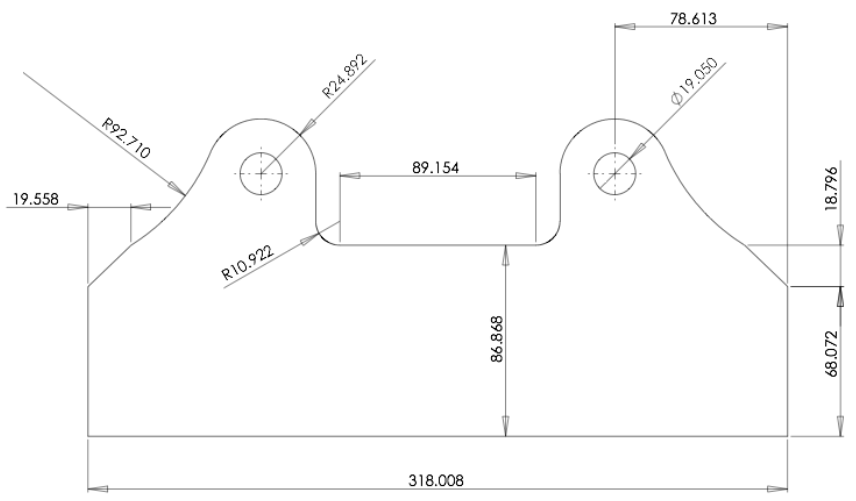

Fig 17. Dimensions of the lug joint (inches)

The lug joint was cyclically loaded with a maximum load of $13 \mathrm{kN}$ and load ratio of 0.1 , at a frequency of $5 \mathrm{~Hz}$. To track the crack growth, two cameras were mounted on to the frame, each focusing on the crack on the front and rear of the specimen. The captured images were used to calculate the crack length. The crack tip locations at different number of cycles are given in Table 1.

Table 1. Crack tip locations at different instances

\begin{tabular}{ccc}
\hline \hline Cycles & Crack tip location $(\mathbf{x}, \mathbf{y})(\mathbf{m m})$ & Crack Length $(\mathbf{m m})$ \\
\hline 90457 & $(109.46,83.8)$ & 5.15 \\
91585 & $(108.60,79.72)$ & 7.44 \\
92883 & $(108.54,79.64)$ & 7.63 \\
93057 & $(108.45,77.08)$ & 10.31 \\
94201 & $(108.14,75.28)$ & 12.02 \\
94384 & $(107.90,73.80)$ & 12.56 \\
94548 & $(107.90,73.59)$ & 13.32 \\
94716 & $(107.42,71.68)$ & 15.47 \\
95014 & $(107.62,70.99)$ & 15.88 \\
95287 & $(106.94,68.68)$ & 18.32 \\
95387 & $(106.94,67.36)$ & 18.47 \\
95439 & $(106.62,66.20)$ & 21.09 \\
95663 & $(106.50,63.33)$ & 21.57 \\
\hline \hline
\end{tabular}




\section{D1. Evaluating SIF as a function of crack tip location:}

In order to evaluate the SIF, a quasistatic finite element simulation of the three dimensional lug joint was run using ABAQUS/Standard [42]. The crack propagation direction was considered as normal to the crack front plane for the SIF calculation.
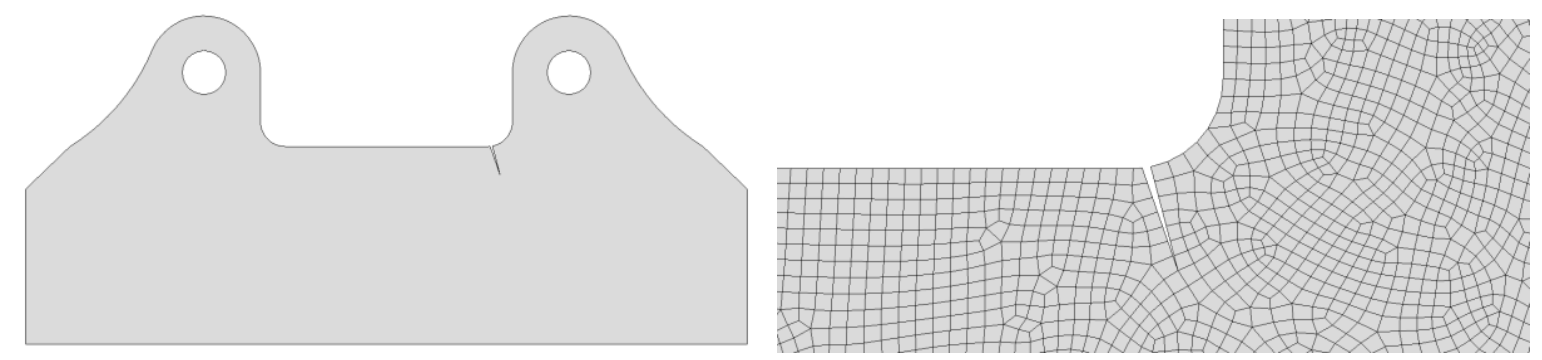

Fig. 18 Finite element model of the lug joint with crack

A grid of $15 \mathrm{~mm} \times 25 \mathrm{~mm}$ was made at one shoulder of the lug joint, and the crack tip was modeled for 17 different locations on the grid. The left pinhole on the lug joint was fixed in all directions, and the right pinhole was allowed to move along the direction of the loading. The SIF was calculated with a load of $13 \mathrm{kN}$. It was also observed that the SIF varies linearly with loading. Table 2 shows the SIF for two additional load cases $(7.5 \mathrm{kN}$ and $3.25 \mathrm{kN})$.

Table 2. SIF for different crack tip locations and loads

\begin{tabular}{ccc}
\hline \hline Crack tip $(\mathbf{x}, \mathbf{y})(\mathrm{mm})$ & Load $(\mathrm{kN})$ & SIF $(\mathrm{Pa} \sqrt{\mathrm{mm}})$ \\
\hline$(109.00,79.50)$ & 13 & $1.08 \mathrm{E}+07$ \\
$(109.00,79.50)$ & 1.3 & $1.08 \mathrm{E}+06$ \\
$(104.00,79.50)$ & 13 & $1.35 \mathrm{E}+07$ \\
$(104.00,79.50)$ & 7.5 & $6.75 \mathrm{E}+06$ \\
$(113.16,84.53)$ & 13 & $2.58 \mathrm{E}+06$ \\
$(113.16,84.53)$ & 3.25 & $6.45 \mathrm{E}+05$ \\
\hline \hline
\end{tabular}

From Table 2 it can be seen that for the first crack tip location $(109.00,79.50)$, as the load reduces 10 times, the SIF also reduces to $1 / 10^{\text {th }}$ of the original value. For the crack tip location $(104.00,79.50)$, as the load reduces by $1 / 2$, the SIF also reduces by $1 / 2$. And, for the crack tip 
location $(113.16,84.53)$, as the load reduces by $1 / 4^{\text {th }}$, the SIF also reduces by $1 / 4^{\text {th }}$. These observations indicate that the SIF varies linearly with the load. Hence, for any given loading, the SIF can be calculated as a linear function of the load. In our experiments, the SIF (K) was calculated for unit load. Then, for any given loading, the calculated SIF was multiplied by the load to get the new SIF for that particular load. From this process, we were able to find the SIF as a function of the crack tip location for different loads. After the SIF was evaluated, a mapping for the SIF was created as a function of the crack tip location. Once the mapping was created, the SIF can be evaluated from the mapping for any obtained crack tip location during the experiment. To create a mapping of the SIF, three methods were used. First, the mapping was created using the surface fitting toolbox in MATLAB [43], and then using LASSO, and then using RVM. The first two methods are deterministic, whereas RVM is a probabilistic method, which provides the confidence intervals of the prediction.

The SIF for different crack tip locations for a load of $13 \mathrm{kN}$ are listed in Table 3.

Table 3. SIF calculated from FEM for different crack tip locations

\begin{tabular}{cc}
\hline \hline Crack Tip $(\mathbf{x , y})$ & SIF $(\mathbf{P a} \sqrt{\mathbf{m m})}$ \\
\hline$(109.00,79.50)$ & $1.08 \mathrm{E}+07$ \\
$(109.00,74.50)$ & $1.09 \mathrm{E}+07$ \\
$(108.71,69.05)$ & $1.39 \mathrm{E}+07$ \\
$(109.00,64.50)$ & $1.85 \mathrm{E}+07$ \\
$(109.00,59.50)$ & $2.45 \mathrm{E}+07$ \\
$(104.00,79.50)$ & $1.35 \mathrm{E}+07$ \\
$(104.00,74.50)$ & $1.47 \mathrm{E}+07$ \\
$(104.00,69.50)$ & $1.83 \mathrm{E}+07$ \\
$(104.00,64.50)$ & $2.06 \mathrm{E}+07$ \\
$(104.00,59.50)$ & $2.24 \mathrm{E}+07$ \\
$(99.00,74.50)$ & $2.16 \mathrm{E}+07$ \\
$(99.00,69.50)$ & $1.89 \mathrm{E}+07$ \\
$(99.00,64.50)$ & $2.05 \mathrm{E}+07$ \\
$(99.00,59.50)$ & $2.48 \mathrm{E}+07$
\end{tabular}




\section{D2. SIF mapping using LASSO:}

To create the mapping for the SIF, the input variables were the crack tip coordinates $x$ and $y$, and the output variable was the SIF. There are 17 data points (i.e., 17 pairs of $\mathrm{x}$ and $\mathrm{y}$ ), and one value of SIF for each data point. The fit between these variables was done using different types of kernels, and the results are shown in the plots below.

\section{Exponential kernel:}

The exponential kernel is a radial basis function kernel, and is closely related to the Gaussian kernel, with the only difference being that there is no square of the norm.

$$
k(x, y)=\exp \left(\frac{\|x \quad y\|}{2^{2}}\right),
$$

where $\quad{ }^{2}$ is the variance of the distribution

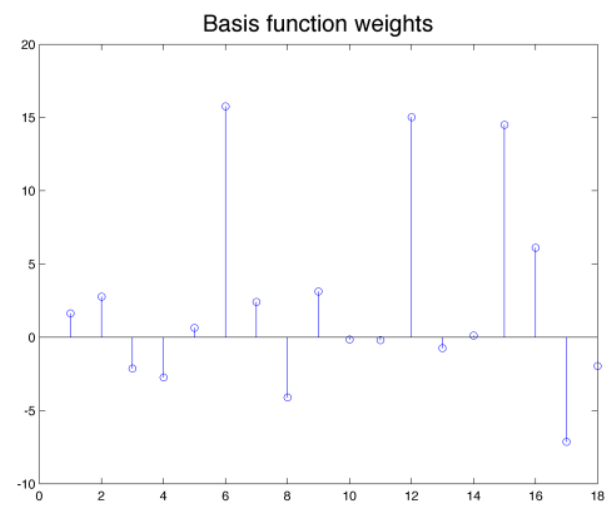

Fig 19. The weights used for each of the basis functions

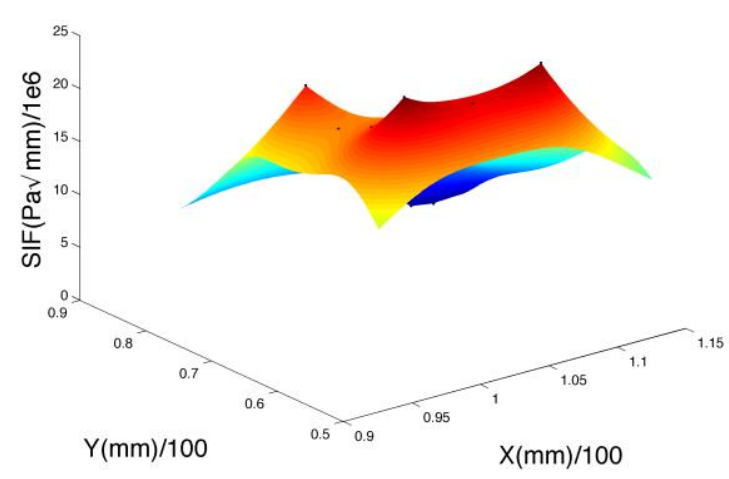

Fig 20. Obtained fit using exponential kernel 


\section{Gaussian kernel:}

The Gaussian kernel is also an example of the radial basis function kernel.

$k(x, y)=\exp \left(\frac{\|x y\|^{2}}{2^{2}}\right)$

where ${ }^{2}$ is the variance of the Gaussian distribution

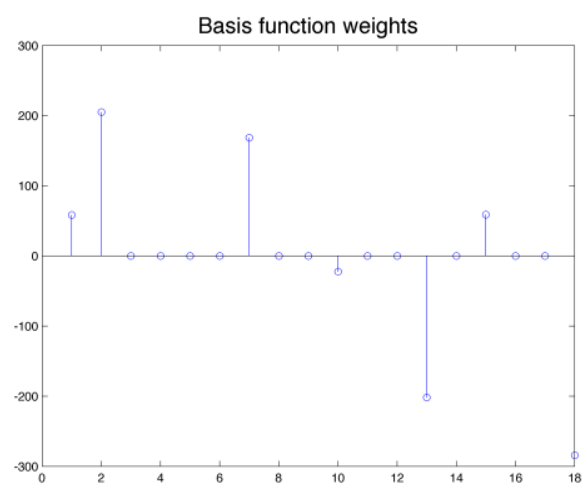

Fig 21. Weights used for each of the basis functions

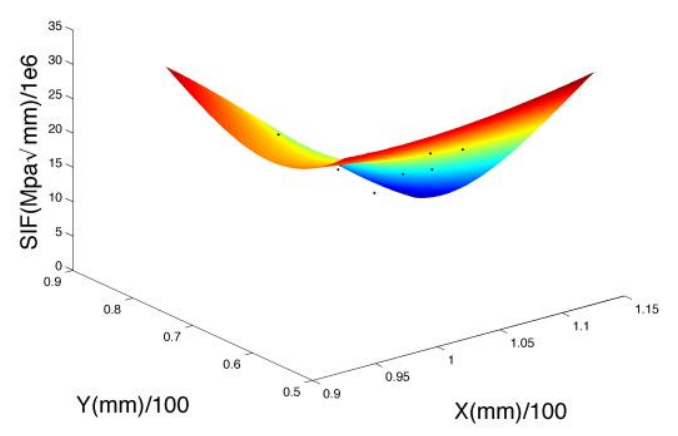

Fig 22. Obtained fit using Gaussian Kernel

\section{Combination of Gaussian and exponential kernels}

Sometimes, using a single kernel may not yield good results no matter how much the parameters of kernels are optimized. In such cases, a linear combination of the kernels can be used.

$k=k_{\text {Gaussian }}+k_{\text {exponential }}$

$k(x, y)=\exp \left(\frac{\|x \quad y\|^{2}}{2^{2}}\right)+\exp \left(\frac{\|x \quad y\|}{2^{2}}\right)$ 


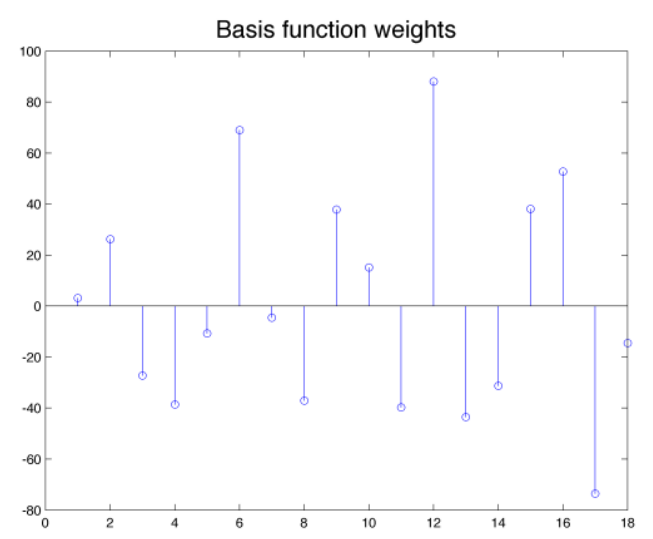

Fig 23. Weights used for each of the basis functions

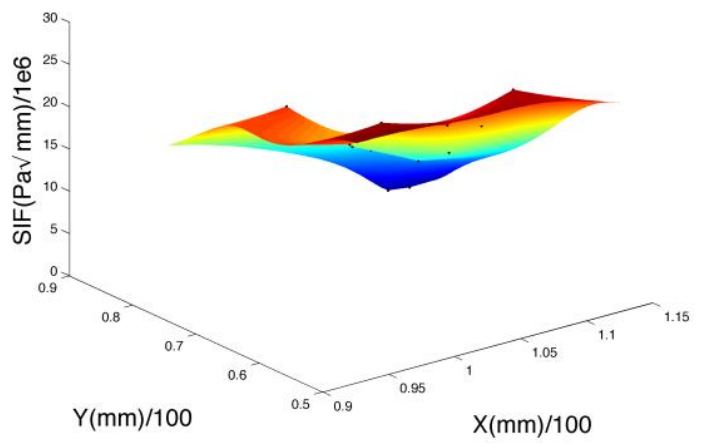

Fig 24. Obtained fit using Gaussian Kernel

It can be seen that using a combination of kernels produces a very good fit for the data.

\section{D3. SIF mapping using RVM}

The SIF is mapped using RVM as described in the equations above. The input parameters are the crack tip coordinates $(\mathrm{x}, \mathrm{y})$, and the output parameter is the SIF for a given crack tip location. The data from Table 3 was used to create the mapping.

While the LASSO is a useful tool for robust regression, it yields a point estimate for the regressed model. RVM is a powerful technique that can perform full probabilistic regression and it has the advantage of additionally providing a measure of uncertainty (confidence intervals) on the regressed estimate. Thus, using the RVM for the SIF mapping, a probability distribution on the SIF as a function of $\mathrm{x}$ and $\mathrm{y}$ position can be obtained. In this setting, the uncertainty in SIF can be transferred naturally to uncertainty in crack growth rate, for subsequent use in the prognosis model.

In our evaluation, the inputs used included 17 data points, and the predictions were plotted on a grid of 201 by 301 for a total of 60501 data points. For each of the 60501 grid 
points, a lower bound and an upper bound were established, which can be related to the variance of the prediction.

The input data was mapped with RVM using a Gaussian kernel, and the obtained fit, with lower bound and upper bound surfaces, is shown in Fig. 25 and Fig. 26. The vertical lines on the surface show the $95 \%$ confidence bounds of the predicted SIF using RVM. The surfaces were plotted with a variance of $2 \sigma$. For this set of input and output data, Gaussian kernel was observed as providing good results; hence this kernel was used for the mapping.

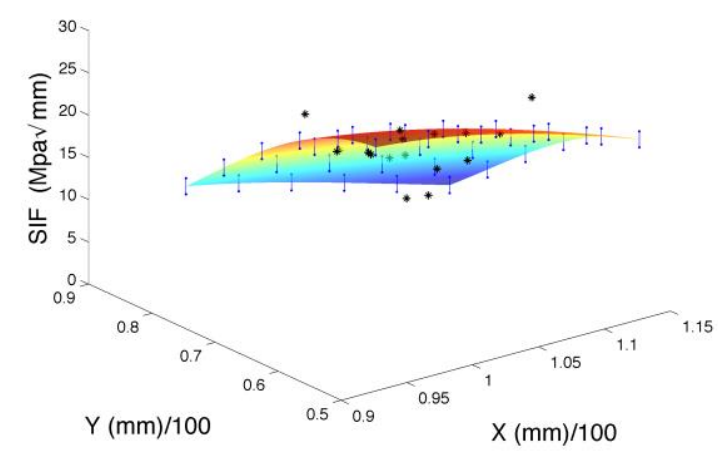

Fig 25. Plot of surface fit using RVM

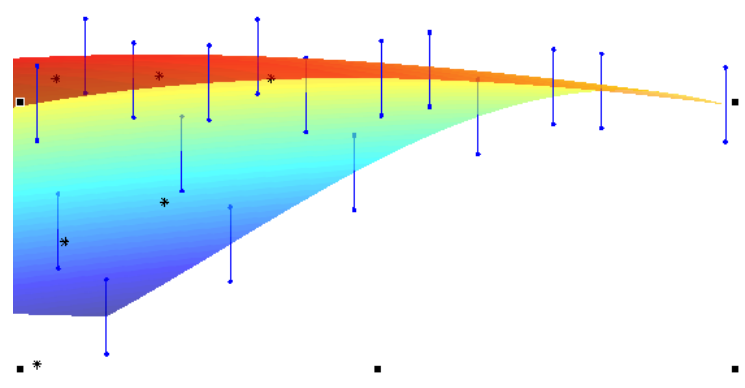

Fig 26. Enlarged view of Fig. 20

\section{E. Prediction using hybrid prognosis:}

The future crack length at any given cycle was first predicted using a deterministic regression. First, the SIF was mapped using a basic surface fitting toolbox in MATLAB. Then, as the experiment continued and the crack tip location was identified, the SIF was determined for the obtained crack tip location from the mapping created. Then, Eq. 2 was used to evaluate the crack growth rate at any given instant, and Eq. 5 was used to predict the future crack length at any given number of cycles. 


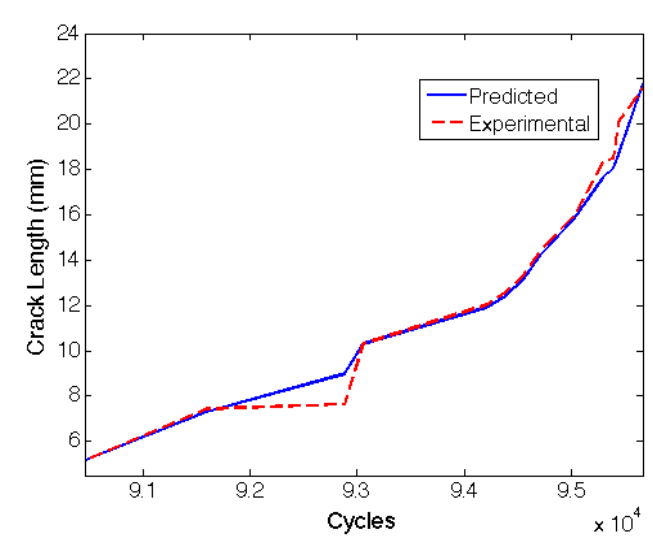

Fig.27 Prediction of the crack length

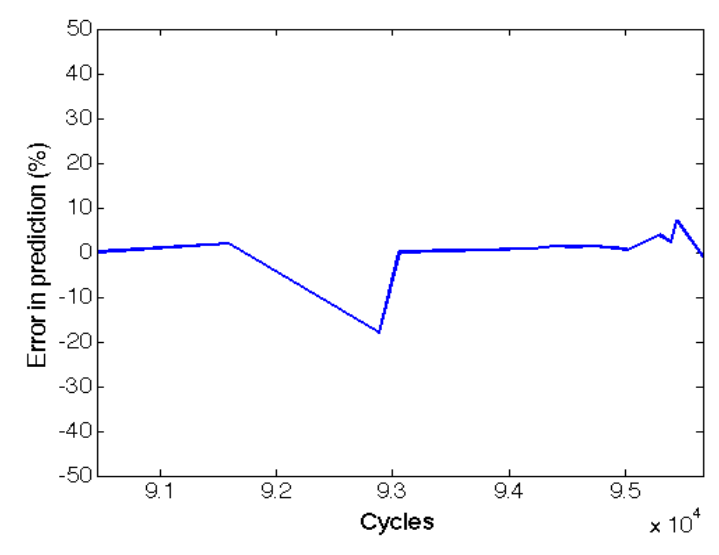

Fig. 28 Error in prediction of crack length

The crack length at $93 \mathrm{k}$ cycles was not calculated properly due to the problem with camera image. Nonetheless, the prognosis model was able to predict the crack length within an error of less than $7 \%$ for almost the entire crack growth regime.

The prediction of the crack length is done in a probabilistic framework, which offers the confidence of prediction. The uncertainty in the prediction of the crack length, however, arises from uncertainty in the prediction of the SIF. When the SIF is evaluated using RVM, we can arrive at the variance in the prediction, while evaluating the mean value at the test point. This variance translates into the confidence in the prediction of the crack length. Fig. 29 shows the prediction with confidence intervals. 


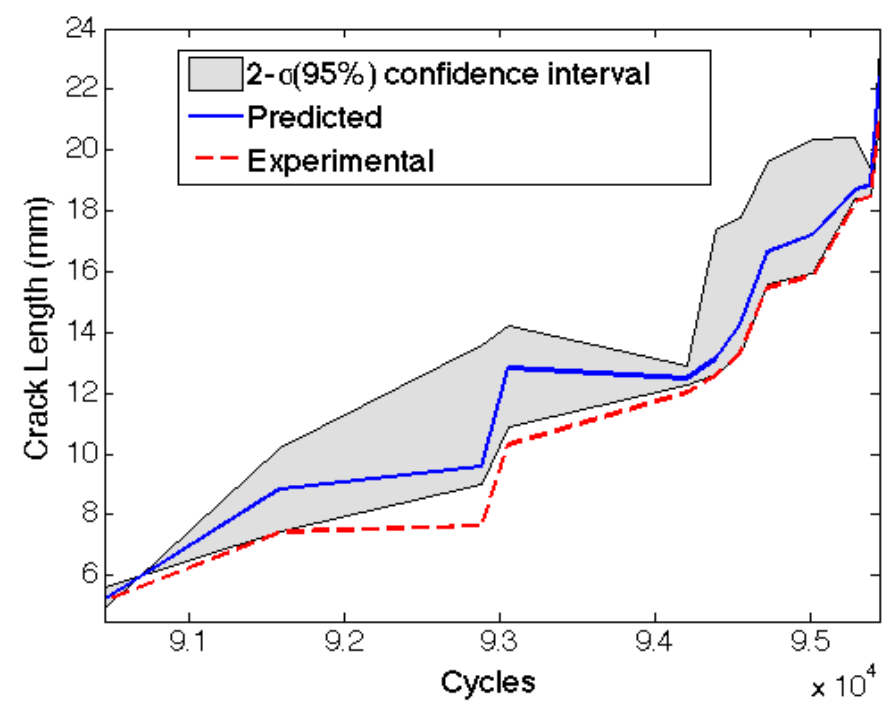

Fig. 29 Prediction with confidence intervals

From Fig. 29 we can see that the algorithm is able to predict the RUL within 2 interval, which translates to $95 \%$ confidence in prediction. The experimental crack length is very close to the lower bound of the $95 \%$ confidence interval. This implies that we are overpredicting the crack length within the $95 \%$ confidence interval which is a fail-safe prediction.

\section{F. Comparison with Paris Law:}

In order to validate the developed hybrid prognosis model, the results obtained from section E are compared with that of classical Paris Law. The SIF obtained from the mapping of SIF using RVM (Section D3) was used to calculate the crack growth rate using Paris law. The crack growth rate at any given cycle was calculated using Eq. 22 as,

$\frac{d a}{d N}=C(K)^{m}$

where, C \&m are the Paris' law constants. As the material used was Al2024-T351, the coefficients $C$ and $m$ are $3.3 \mathrm{e}-10$ and 2.3 respectively. Using these coefficients and the SIF obtained using RVM, the crack growth rate was calculated at any given cycle. Once the crack 
growth rate $(\mathrm{da} / \mathrm{dN})$ is obtained at a cycle $(\mathrm{N})$, the future crack length at cycle $(\mathrm{N}+\Delta \mathrm{N})$ can be calculated as:

$\mathrm{a}_{\mathrm{N}+\Delta \mathrm{N}}=\mathrm{a}_{\mathrm{N}}+(\mathrm{da} / \mathrm{dN}) * \Delta \mathrm{N}$,

where $a_{N}$ is the crack length at the current cycle.

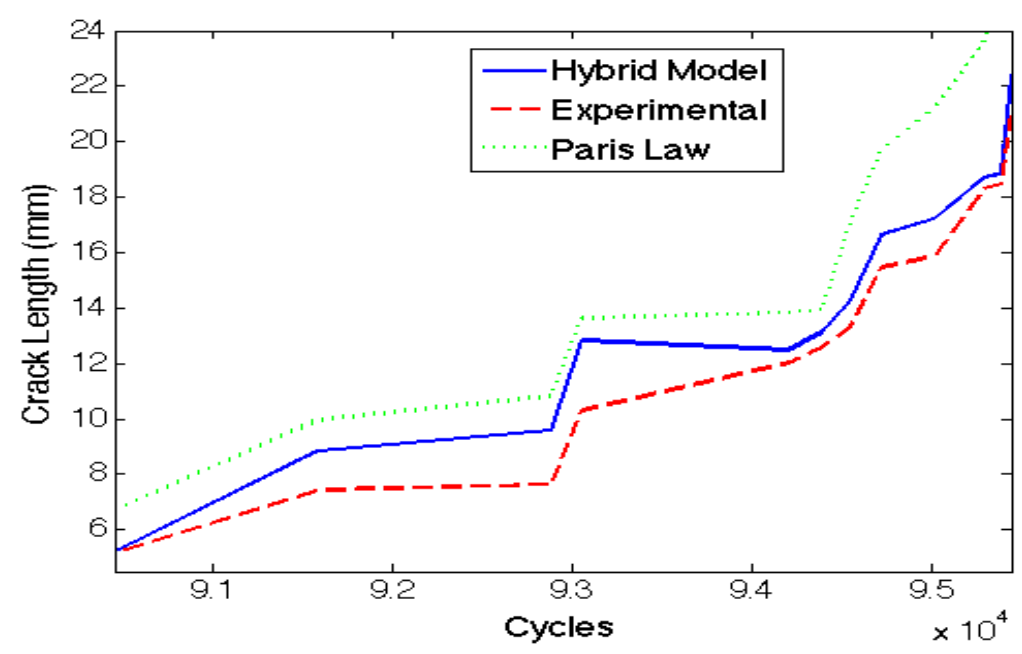

Fig. 30 Comparison between hybrid prognosis model and Paris’ Law

Fig. 30 shows the comparison of the predicted crack lengths using the hybrid model and Paris law. The crack length predicted using Paris law is highly over-predicted as shown in Fig. 30. Whereas, the crack length predicted by the hybrid model is over-predicted only within the 95\% confidence interval (Fig. 29), which is the best possible scenario for any practical application. It is to be noted that the result of the crack length predicted using Paris' law would not always be over-predicted. For this particular case study, the crack length was over-predicted.

\section{Conclusions}

A hybrid prognosis methodology has been developed integrating a simple physics based approach with experimental data. The algorithm provides high fidelity predictions of RUL for CT specimens subject to various loading conditions. Linear fit models were used to extrapolate and predict the crack growth behavior. The RUL prediction is within $\pm 5 \%$ of the actual RUL for 
constant amplitude loading. The methodology was applied to random loading conditions; the mean of the random data set was used as the initial training data. The RUL prediction was within $\pm 5 \%$ for the random loading case, reducing to $\pm 2 \%$ as the training data increased. The algorithm was modified to incorporate the crack closure phenomenon observed during an overload. Once again, an error of $\pm 5 \%$ was observed if the assumed point of overloads was known. However, even if the overload points were unknown, the algorithm was still able to predict within 5\% error given training data for overload behavior. The algorithm was then validated with the experimental data from the fatigue test conducted on an Al 2024-T351 lug joint. The algorithm was able to predict the crack length at any given instant with an error of less than $7 \%$ for most of the crack growth regime. The proposed methodology was compared with Paris' law and the current method gives more accurate results with high confidence.

\section{Acknowledgements}

This research is supported by the U.S. Department of Defense, U.S. Air Force Office of Scientific Research Multidisciplinary University Research Initiation Grant, FA95550-06-1-0309, Technical Monitor: David Stargel.

\section{References}

${ }^{1}$ Newman, J.C.Jr. "FASTRAN-II - A Fatigue Crack Growth Structural Analysis Program. NASA Technical Memoradum 104159, Langley Research Center, 1992.

${ }^{2}$ Newman J.C. Jr., “A crack-opening stress equation for fatigue crack growth”, International Journal of Fracture, Vol 24, 1984, pp.131-135. 
${ }^{3}$ AFGROW 4.0004.12.10, AFRL/VASM, 2001.

${ }^{4}$ Newman, J.C., Jr. 1982, "Prediction of fatigue crack growth under variable-amplitude and spectrum using a closure model", ASTM STP 761: 255-277.

${ }^{5}$ Ray, A., and Patankar, R., "Fatigue crack growth under variable amplitude loading: Part I Model formulation in state-space setting", Applied Mathematical Modeling, Vol 25, 2001, pp. 979-994.

${ }^{6}$ Ray, A., and Patankar, R., "Fatigue crack growth under variable amplitude loading: Part II Code development and model validation", Applied Mathematical Modeling, Vol 25, 2001, pp. 995-1013.

${ }^{7}$ Zapateroa, J. and Domnguez, J., "A statistical approach to fatigue life prediction under random loading", International Journal of Fatigue, Vol. 12(2), 1990, pp . 107-114.

${ }^{8}$ Liu, Y. and Sankaran, M., "Stochastic fatigue damage modeling under variable amplitude loading", International Journal of Fatigue, Vol. 29(6), 2007, pp. 1149-1161.

${ }^{9}$ Ling, Y., and Mahadevan, S., "Integration of structural health monitoring and fatigue damage prognosis", Mechanical Systems and Signal Processing, Vol 28, 2012, pp. 89-104. 
${ }^{10}$ Ling, Y., Shantz, C., Mahadevan, S., and Sankararaman, S., "Stochastic prediction of fatigue loading using real-time monitoring data", International Journal of Fatigue, Vol 33, 2011, pp. 868-879.

${ }^{11}$ Sankararaman, S., Ling, Y., Shantz, C., and Mahadevan, S., "Uncertainty Quantification in Fatigue Damage Prognosis", Annual Conference of the Prognostics and Health Management Society, 2009.

${ }^{12}$ Liu, Y. and Mahadevan,S., "Probabilistic fatigue life prediction using an equivalent initial flaw size distribution", International Journal of Fatigue, Vol. 31, 2009, pp. 476-487.

${ }^{13} \mathrm{Hu}$, W.P., Shen, Q. A., Zhang, M., Meng, Q. C. and Zhang, X., "Corrosion-fatigue life prediction for 2024-T62 aluminum alloy using damage mechanics-based approach", International Journal of Damage Mechanics, Vol. 21, 2012, pp. 1245-1266.

14 Grell, W. A. and Laz, P. J., "Probabilistic fatigue life prediction using AFGROW and accounting for material variability", International Journal of Fatigue, Vol. 32, 2010, pp. 10421049.

15 Ozaltun, H., Shen, M. -H. H., George, T. and Cross, C., “ An energy based fatigue life prediction framework for in-service structural components", Society for Experimental Mechanics, Vol. 51, 2011, pp. 707-718. 
${ }^{16}$ Newman, Jr. J. C., “A crack-closure model for predicting fatigue crack growth under aircraft spectrum loading”, ASTM STP 748, 1981, pp.53-84

${ }^{17}$ Schijve, J., Skorupa, M., Skorupa, A., Machinewicz, T., and Gruszczynski, P., "Fatigue crack growth in the aluminium alloy D16 under constant and variable amplitude loading", International Journal of Fatigue, Vol 26, 2004, pp. 1-15.

${ }^{18}$ Kermanidis, Al, Th., and Pantelakis, Sp. G., "Fatigue crack growth analysis of 2024 T3 alumunium specimens under aircraft service spectra", 2001 Blackwell Science Ltd. Fatigue Fract Engng Mater Struct, Vol 24, pp. 699-710.

${ }^{19}$ Xiang, Y., Lu, Z., and Liu, Y., “Crack growth-based fatigue life prediction using an equivalent initial flaw model. Part I: Uniaxial Loading”, International Journal of Fatigue, Vol 32(2), 2010, pp. 376-381.

${ }^{20}$ Harter, J.A. 1999, “AFGROW Users’ Guide and Technical Manual. Report No. AFRL-VAWP-1999-3016", Air Force Research Laboratory.

${ }^{21}$ NASGRO v3.0.20, May 2002.

${ }^{22}$ Mohanty, S., Chattopadhyay, A., and Peralta, P., "Adaptive Residual Useful Life Estimation of a Structural Hotspot”, Journal of Intelligent Material Systems and Structures, Vol. 21, February 2010. 
${ }^{23}$ Liu, Y., Mohanty, S. and Chattopadhyay, A., "Condition based structural health monitoring and prognosis of composite structures under uniaxial and biaxial loading," Journal of Nondestructive Evaluation, Vol. 29, No. 3, 2010, pp. 181-188, 2010.

${ }^{24}$ Mohanty, S., Chattopadhyay, A., Peralta, P.,and Quech, D., "Fatigue damage prognosis of a cruciform structure under biaxial random and flight profile loading", Nondestructive characterization for composite materials, Aerospace Engineering, Civil Infrastructure and Homeland Security 2010, Proceedings of SPIE, Vol. 7649

${ }^{25}$ Chattopadhyay, A., and Mohanty, S., "Gaussian Process Damage Prognosis under Random and Flight Profile Fatigue Loading", Machine Learning and Knowledge Discovery for Engineering Systems Health Management, Chapman and Hall / CRC 2011, Chapter 6, pp. 181202.

${ }^{26}$ Mohanty, S., Chattopadhyay, A. and Rajadas, J.N., "Dynamic strain mapping and real-time damage-state estimation under random fatigue loading”, AIAA Journal, Vol. 50.4, 2012, pp.769777.

${ }^{27}$ Wang, P., Youn, B. D. and Chao, H., "A generic probabilistic framework for structural health prognostics and uncertainty management”, Mechanical Systems and Signal Processing, Vol. 28, 2012, pp. 622-637. 
${ }^{28}$ Gebraeel, N. and Pan, J., "Prognostic degradation models for computing and updating residual life distributions in a time-varying environment", IEEE Transactions on Reliability, Vol. 57(4), 2008, pp. 539-550.

${ }^{29}$ Bian, L. and Gebraeel, N., " Computing and updating the first-passage time distribution for randomly evolving degradation signals”, IIE Transactions, Vol. 44, 2012, pp. 974-987.

${ }^{30}$ Gebraeel, N., Elwany, A. and Pan, J., "Residual life predictions in the absence of prior degradation knowledge", IEEE Transactions on Reliability, Vol. 58(1), 2009, pp. 106-117.

${ }^{31}$ Suresh S. "Fatigue of Materials", Cambridge University Press, Cambridge, U.K. 1991.

${ }^{32}$ Soni, S. O., Kim, S. B. and Chattopadhyay, A., "Fatigue crack detection and localization using reference-free method", Proceedings of SPIE 7648, 2010, Paper number: 76480U.

${ }^{33}$ Hensberry, K., Kovvali, N., Liu, K. C., Chattopadhyay, A. and Papandreou-Suppappola, A., "Guided wave based fatigue crack detection and localization in aluminum aerospace structures", Proceedings of the 2012 ASME Conference on Smart Materials, Adaptive Structures and Intelligent Systems (SMASIS), 2012, Paper Number: 8241

34 Tibshirani, Robert., "Regression shrinkage and selection via the lasso", Journal of the Royal Statistical Society, Series B(Methodological), Vol. 58, No. 1 (1996), pp 267-288. 
${ }^{35}$ Tipping, Michael E., "Sparse Bayesian Learning and the Relevance Vector Machine”, Journal of Machine Learning Research, Vol. 1, 2001, pp. 211-244.

${ }^{36} \mathrm{Wu}, \mathrm{W} . \mathrm{F}$., and Ni, C.C., "Probabilistic models of fatigue crack propagation and their experimental verification”, Probabilistic Engineering Mechanics Vol 19, 2004, pp. 247-257.

${ }^{37}$ ASTM standard E647-93, Standard test method for measurement of fatigue crack growth rates, Am Soc Test Mater 1994;03.01:679-706.

${ }^{38}$ McMaster, F.J., and Smith, D.J., "Predictions of fatigue crack growth in aluminium alloy 2024-T351 using constraint factors”, International Journal of Fatigue, Vol 23, 2001, S93-S101.

${ }^{39}$ Murakami, Y., Stress Intensity Factors Handbook, The Soc. Material Science, Japan \& Elsevier Science Ltd, Vol. 1\&2.

${ }^{40}$ Carlson, R.L., Kardomateas, G.A. and Bates, P.R., "The effects of overloads in fatigue crack growth", International Journal of Fatigue, Vol.13, No. 6, 1991, pp. 453-460.

${ }^{41}$ Abaqus Version 6.7, Abaqus/CAE and Abaqus/Explicit. 2007. Simulia World Headquarters, Providence, United States.

${ }^{42}$ MATLAB and Statistics Toolbox Release 2012b, The Mathworks, Inc., Natick, Massachusetts, United States. 